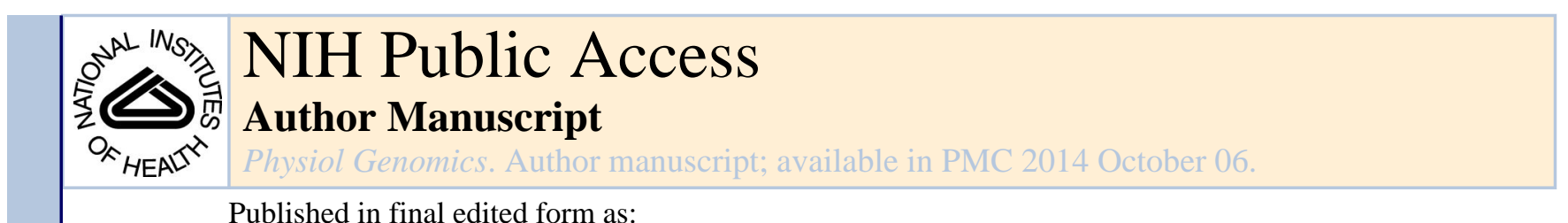

Published in final edited form as:

Physiol Genomics. 2007 August 20; 30(3): 282-299. doi:10.1152/physiolgenomics.00242.2006.

\title{
Microarray analysis of the temporal response of skeletal muscle to methylprednisolone: comparative analysis of two dosing regimens
}

\author{
Richard R. Almon ${ }^{1,2}$, Debra C. DuBois ${ }^{1,2}$, Zhenling Yao ${ }^{1}$, Eric P. Hoffman ${ }^{3}$, Svetlana \\ Ghimbovschi ${ }^{3}$, and William J. Jusko ${ }^{1}$ \\ ${ }^{1}$ Department of Pharmaceutical Sciences and State University of New York (SUNY) at Buffalo, \\ Buffalo, New York \\ ${ }^{2}$ Department of Biological Sciences, State University of New York (SUNY) at Buffalo, Buffalo, \\ New York \\ ${ }^{3}$ Children's National Medical Center, Washington, District of Columbia
}

\begin{abstract}
The transcriptional response of skeletal muscle to chronic corticosteroid exposure was examined over $168 \mathrm{~h}$ and compared with the response profiles observed following a single dose of corticosteroid. Male adrenalectomized Wistar rats were given a constant-rate infusion of 0.3 $\mathrm{mg} \cdot \mathrm{kg}^{-1} \bullet \mathrm{h}^{-1}$ methylprednisolone for up to 7 days via subcutaneously implanted minipumps. Four control and forty drug-treated animals were killed at ten different time points during infusion. Liver total RNAs were hybridized to 44 individual Affymetrix REA230A gene chips. Previously, we described a filtration approach for identifying genes of interest in microarray data sets developed from tissues of rats treated with methylprednisolone (MPL) following acute dosing. Here, a similar approach involving a series of three filters was applied sequentially to identify genes of interest. These filters were designed to eliminate probe sets that were not expressed in the tissue, not regulated by the drug, or did not meet defined quality control standards. Filtering eliminated $86 \%$ of probe sets, leaving a remainder of 2,316 for further consideration. In a previous study, 653 probe sets were identified as MPL regulated following administration of a single (acute) dose of the drug. Comparison of the two data sets yielded 196 genes identified as regulated by MPL in both dosing regimens. Because of receptor downregulation, it was predicted that genes regulated by receptor-glucocorticoid response element interactions would exhibit tolerance in chronic profiles. However, many genes did not exhibit steroid tolerance, indicating that present perspectives on the mechanism of glucocorticoid action cannot entirely explain all temporal profiles.
\end{abstract}

\section{Keywords}

glucocorticoids; corticosteroids; Affymetrix gene chips; gene expression; time series

Copyright (C) 2007 the American Physiological Society

Address for reprint requests and other correspondence: R. Almon, Dept. of Biological Sciences, 107 Hochstetter Hall, SUNY at Buffalo, Buffalo, NY 14260 (almon@eng.buffalo.edu).. 
Glucocorticoids are a class of steroid hormones that play a central role in regulating the production, storage, use, and distribution of substrates for systemic energy metabolism. Most tissues are targets for glucocorticoid action and contribute in some way to their wideranging physiological effects. Synthetic glucocorticoids (corticosteroids) are used therapeutically for a wide variety of conditions that require immune and/or inflammatory modulation, including the treatment of muscular dystrophy. Because corticosteroids pharmacologically magnify the physiological actions of glucocorticoids, therapeutic use of this class of drugs is accompanied by a wide range of adverse effects including muscle wasting, hyperglycemia, dyslipidemia, hypertension, nephropathy, fatty liver, and an increased risk of arteriosclerosis $(12,20,30,37,45)$. The musculature contributes significantly to several of these adverse effects. Corticosteroids shift the musculature into a net negative nitrogen balance, causing muscle wasting. The resultant amino acid carbon is released into circulation primarily in the form of glutamine and is taken up by the liver and kidney for use as a substrate for gluconeogenesis. The glucose is released into circulation, causing secretion of insulin. However, because corticosteroids cause insulin resistance and skeletal muscle is responsible for $\sim 80 \%$ of the insulin-directed glucose disposal, the result is hyperglycemia (10). In addition, increased resistance in the muscle vasculature caused by corticosteroids contributes to hypertension (28).

The physiological and pharmacological effects of these compounds are complex and involve changes in the expression of many genes in multiple tissues. However, changes in expression of a single gene may involve primary and/or higher-order levels of control. A primary response can be considered as the direct transcriptional modulation of that gene by a steroid-bound receptor. Higher-order regulation may involve alteration of a secondary biosignal (which may include alteration of the expression or action of other transcription factors, either directly or indirectly, that modulate expression of that gene). Understanding the complexity of gene expression changes is a necessary first step to a comprehensive understanding of how such changes translate into altered systemic physiology and contribute to the adverse effects of corticosteroids. Within a single tissue, it is necessary to identify those genes that are regulated by steroid treatment as well as the temporal patterns of those changes following treatment.

Microarrays can provide a method for the high-throughput data collection that is necessary for constructing a comprehensive view of the transcriptional basis of such complex systemic polygenic phenomena. When microarrays are used in a rich in vivo time series, they yield temporal patterns of changes in gene expression that illustrate the cascade of molecular events in time that cause broad, complex responses. However, the magnitude of data produced in such studies provides challenges of data mining and analysis.

Previously, we described the mining and analysis of microarray time series, illustrating the responses of skeletal muscle, liver, and kidney taken from the same set of animals to a single bolus dose of the corticosteroid methylprednisolone (MPL) $(4,6,7)$. Those studies included individual chips from multiple control animals as well as multiple animals at each of 16 times over a 72-h period following acute dosing with MPL. Because those experiments were initiated using adrenalecto mized animals, the drug in effect acted as a stimulus that 
perturbed the homeostatic balance of the system, and the experiment monitored the deviation of the system and its return to the original state.

Although very useful, a single-dose time series only provides a limited view of the dynamics of the system in response to the drug. A pharmacological time series is different from most time series studies (for example, those assessing developmental changes) in that it can be repeated using different dosing regimens. Additional dosing regimens are valuable in that they can illustrate response profiles of genes to different patterns of input perturbations. Two genes may respond with the same temporal profile to a single-dosing regimen, suggesting that their mechanism of regulation is the same. However, they may not respond to a second dosing regimen with the same response profile, demonstrating that their mechanisms of regulation are in fact different. The results from multiple dosing regimens can therefore be used to group genes into clusters with common mechanisms of regulation. The predicating assumption is that if two or more genes have a common mechanism of regulation, then their response profiles should be the same regardless of the dosing regimen.

In the present report, we describe the use of microarrays to broadly characterize the response of skeletal muscle to both single-dose and chronic infusion of MPL. In the latter, the drug essentially serves as an unbalancing stimulus, and the experiment evaluated the capacity of the system to rebalance in the continuous presence of the drug. This data set was mined using a similar filtration approach as was applied to the acute-dosing data set, and results from both data sets were compared. Probe sets common to both filtrations were identified, allowing the coincidental evaluation of the two profiles for each gene. Examination of acute and chronic profiles clearly illustrates that corticosteroids influence the expression of genes through a wide variety of mechanisms ranging from the relatively simple, in which the chronic profile shows tolerance because of the downregulation of the glucocorticoid receptor, to very complex patterns that not only do not show tolerance but also show patterns suggesting an interplay of multiple mechanistic influences.

\section{MATERIALS AND METHODS}

\section{Animals}

Adrenalectomized male Wistar rats with body weights of $339-28$ (SD) g were used in the study. All animals were housed in our University Laboratory Animal Facility and maintained under constant temperature $\left(22^{\circ} \mathrm{C}\right)$ and humidity with a controlled 12:12-h lightdark cycle. A time period of at least 2 wk was allowed before animals were prepared for surgery. Rats had free access to rat chow and $0.9 \% \mathrm{NaCl}$ drinking water. This research adheres to Principles of Laboratory Animal Care (National Institutes of Health publication no. 85-23, revised 1985) and was approved by the Institutional Animal Care and Use Committee of SUNY at Buffalo.

Forty rats were given infusions $\left(0.3 \mathrm{mg} \bullet \mathrm{kg}^{-1} \bullet \mathrm{h}^{-1}\right)$ of methylprednisolone sodium succinate (Solu-Medrol; The Upjohn, Kalamazoo, MI) reconstituted in supplied diluent. The infusions were administered via Alzet osmotic pumps (model no. 2001, flow rate $1 \mu \mathrm{l} / \mathrm{h}$; Alza, Palo Alto, CA). The pump drug solutions were prepared for each rat based on its predose body weight. On the day of implantation, rats were anesthetized using 60-80 $\mathrm{mg} / \mathrm{kg}$ ketamine and 
8-10 mg/kg xylazine (im). Pumps were subcutaneously implanted between the shoulder blades on the back. Rats were killed at various times, up to 7 days. The time points included were $6,10,13,18,24,36,48,72,96$, and $168 \mathrm{~h}$ after pump implantation. A vehicle-treated control group of four animals was implanted with a saline-filled pump and killed at various times throughout the 7-day study period $(6,18,48$, and $96 \mathrm{~h}$ after pump implantation). Because of the fact that pump implantation requires some time to perform, an actual time zero control is not possible to obtain. Therefore, the earliest experimental time point $(6 \mathrm{~h})$ served as the first control death time, and all controls are considered as nominal time zero. A more detailed description of the experiment can be found in previous reports $(35,36)$.

\section{RNA preparation}

Gastrocnemius muscles from each animal were ground into a fine powder in a mortar cooled by liquid nitrogen, and $100 \mathrm{mg}$ of powder were added to $1 \mathrm{ml}$ of prechilled Trizol Reagent (Invitrogen, Carlsbad, CA). Total RNA extractions were carried out according to the manufacturer's directions and were further purified by passage through RNeasy minicolumns (Qiagen, Valencia, CA) according to the manufacturer's protocols for RNA cleanup. Final RNA preparations were resuspended in RNase-free water and stored at $-80^{\circ} \mathrm{C}$. The RNAs were quantified spectrophotometrically, and purity and integrity were assessed by agarose gel electrophoresis. All samples exhibited 260/280 absorbance ratios of 2.0, and all showed intact ribosomal $28 \mathrm{~S}$ and 18S RNA bands in an approximate ratio of 2:1 as visualized by ethidium bromide staining.

\section{Kinetic-based quantitative RT-PCR}

Kinetic-based RT-PCR assessment of myostatin mRNA was carried out using TaqManbased fluorescent probes in a MX3000 fluorescence-based thermocycler (Stratagene, La Jolla, CA). A one-tube/two-enzyme assay design employing the Brilliant 1-Step Quantitative Core Reagent Kit (Stratagene) was used according to the manufacturer's directions. Concentrations of $\mathrm{MgCl}_{2}$ were $3 \mathrm{mM}$, forward and reverse probe concentrations were $200 \mathrm{nM}$, and probe concentrations were $100 \mathrm{nM}$. Probe and primers were designed using PrimerExpress software (Applied Bio-systems, Foster City, CA), and the sequences used shared no homology with other known rat sequences. Probe and primers were custom synthesized by Biosearch Technologies (Novato, CA) and contained a FAM label on the 5'end and black hole quencher (BHQ1) on the 3'-end. The sequences were as follows: forward primer, 5'-TTGGATGAGAATGGGCATGA-3'; reverse primer, 5'-

ACCTCTTGGGTGTGTCTGTTACT-3'; and probe, 5'-

CTTGCTGTAACCTTCCCAGGACCAGGA-3'. Amplicon length was 103 bp. Signals were quantified against cRNA standards prepared from the myostatin coding sequence cloned into pCR II TOPO vector (Invitrogen) and were prepared by in vitro transcription using T7 Megascript Kits (Ambion, Austin, TX). Samples were normalized to the amount of total RNA in each assay tube. Seven cRNA standards were run concurrently on the same plate in duplicate with tissue RNA samples, which were run in triplicate. RT minus controls were also run on the same plate for each sample to test for possible genomic contamination of RNA samples and in all cases gave no measurable amplification signal. Intra- and interassay coefficients of variation were $<18 \%$. 


\section{Microarrays}

Isolated RNA from each muscle sample was used to prepare the target according to the manufacturer's protocols. The biotinylated cRNAs were hybridized to 44 individual Affymetrix GeneChips, Rat Genome 230A (Affymetrix, Santa Clara, CA), that contained 15,967 probe sets. These gene chips contain over 7,000 more probe sets than the ones used (U-34A) in our previous acute-dose MPL study in muscle (6). The high reproducibility of in situ synthesis of oligonucleotide chips allows accurate comparison of signals generated by samples hybridized to separate arrays.

\section{Data analysis and public access}

All data reported here are available to the public via both the Public Expression Profiling Resource (PEPR) and the National Center for Biotechnology Information (NCBI) Gene Expression Omnibus (GEO), as raw and processed (probe set algorithm) data. In PEPR (http://pepr.cnmcresearch.org), all data are preprocessed into five probe set algorithms [DNA Chip Analyzer (dCHIP) perfect match, dCHIP mismatch model, robust multichip average (RMA), Microarray Suite (MAS)5.0, and ProbeProfiler] and available for dynamic queries. For this present manuscript, we opted to use MAS5.0 probe set algorithms. While other probe set algorithms have been shown to show better performance than MAS5.0 (23, 46), we have made both raw data and five probe set interpretations available to the public. For Affymetrix MAS5.0, signal intensities were normalized for each chip using a distribution of all genes around the 50th percentile. The data set was then loaded into a data mining program, GeneSpring 7.0 (Silicon Genetics, Redwood City, CA). The generated data set has been submitted to the NCBI GEO database (http://www.ncbi.nlm.nih.gov/ projects/ geo/; accession no. GSE5101).

The first step in the analysis involved screening the data from the 11 groups of 4 animals for variance anomalies. This was an important consideration, since the four control animals used in this study were killed at various times $(6,18,48$, and $96 \mathrm{~h})$ within the experimental time frame. This step involved calculation of the mean, standard deviation, and coefficient of variation (CV) of the raw data for each of the 15,967 individual probe sets on the four chips at each individual time point (data from 4 drug-treated animals killed at the same time point) and for the controls (data from 4 vehicle-treated animals killed at 4 different time points). The range of the CVs for each gene at each time point was $40.8-50.2 \%$. The average $\mathrm{CV}$ for the drug-treated samples was $44.8 \%$, and the $\mathrm{CV}$ for the control group was $44.6 \%$.

Before screening for probe sets with altered expression levels, the data set was normalized again to the mean values of control samples so that all probe sets from control samples had a mean value of 1 , and probe sets from treated animals had a value of either greater than, less than, or equal to 1, representing upregulation, downregulation, or no change, respectively. At present, there are a variety of probe set algorithms available to apply to gene array data sets. Most of these are designed to select probe sets that discriminate one group from another. All of these algorithms, MAS5.0, RMA, GC-RMA, and Probe Logarithmic Intensity Error (PLIER), have strengths and weaknesses, and none is designed to take advantage of the unique strengths afforded by a rich time series (38). For our initial analysis

Physiol Genomics. Author manuscript; available in PMC 2014 October 06. 
of this data set, our objective was to select with a very high certainty those probe sets that were regulated by corticosteroids. To accomplish this goal, the normalized probe sets were then filtered with a series of predetermined screening criteria to identify probe sets with appreciable expression levels, expression changes, and acceptable data quality. This set of filters is approximately the same as was used to analyze the acute-dosing data sets with minor modifications to accommodate the different number of samples/chips in the two experiments $(4,6,7)$.

The process of data mining was performed in the GeneSpring program, and the progress after each step was visualized using the gene tree cluster feature of the program. This cluster feature rearranges the order of the probe sets and groups them based on the similarity (Pearson's correlation) of their expression dynamics. Then the probe sets that were not eliminated by the filter were displayed vertically as a gene tree, and their expression dynamics over time were displayed horizontally in colors. Yellow in the graph represents an expression ratio around 1 , or no change. The color progressing toward red indicates a normalized value $>1$, or upregulation, and the color toward blue indicates a value $<1$, or downregulation from control levels. The brightness of the colors reflects the original signal intensities or expression levels before normalization. The more abundantly expressed mRNA exhibit the brighter color. Figure 1 shows the gene tree of the entire data set $(15,967$ probe sets). The $x$-axis represents the 11 time points including vehicle controls (nominally time 0 ). The $y$-axis represents the list of the probe sets in the order of similarity. Figure 2, top, shows a magnification of four probe sets with an apparent response of enhanced expression taken from the tree shown in Fig. 1. A significant deficit of the gene tree representation is that all time intervals are represented as equal, and therefore to some degree temporal patterns are misrepresented. This is illustrated in Fig. 2, bottom, which shows a linear plot of the data for the four probe sets presented at Fig. 2, top. This plot also illustrates the relationship between intensity of color and signal. The lightest probe set in the gene tree is the bottom plot, while the most intensely colored probe set is the top plot.

Similarly, Fig. 3 shows a magnification of five probe sets with apparent downregulation as well as linear plots of these five probe sets. As illustrated in these two examples, it is possible to visually identify genes under regulation using gene trees. However, this approach does not entail objective criteria for selection of probes for further consideration.

To screen for regulated probe sets objectively, the entire data set was filtered with criteria similar to the ones applied to the data set from an acute-dose MPL experiment $(4,6,7)$. This approach does not select for probe sets but rather eliminates those probe sets that do not meet certain criteria, leaving the remainder for further consideration. The first filter was designed to eliminate probe sets for genes that are not expressed in the muscle. This filter enlisted a function in Affymetrix MAS5.0. During initial data analysis, a "call" of present $(\mathrm{P})$, absent $(\mathrm{A})$, or marginal (M) for each probe set on each chip was generated based on the intensity comparison of the matched and mismatched probe sequence pairs. The PM/MM comparison was designed to be used for comparisons at terminal points in time of binary nature (benign vs. malignant, type A vs. type B, etc.) and is the subject of much discussion when considering probe set algorithms (38). We employed the PM/MM comparison in a limited manner as a filter. The first filter is based on the logic that, if the gene is expressed in 
the tissue, then PM should be greater than MM at least on a small percentage of the chips. Therefore, the first filter eliminated all probe sets that did not have a call of $\mathrm{P}$ on at least 3 of the 44 chips (3P). This filter eliminated 6,151 probe sets, leaving a remainder of 9,816 for further consideration. Those probe sets that were not eliminated are presented as a gene tree in Fig. 4. These genes are more likely to be expressed in rat muscle than those that were eliminated.

The second level of filtering was designed to eliminate probe sets that could not meet the basic criterion of a regulated probe. Specifically, this filtering approach eliminates probe sets whose average did not deviate from baseline by a certain value for a reasonable number of time points and employed two filters that were designed to eliminate probe sets that were neither down- nor upregulated. The first of these filters eliminated probe sets that could not meet a minimal criterion for downregulation. Starting with the 3P filtered list, we eliminated all probe sets that did not have average values $<0.65$ in at least two conditions (time points). Those that were not eliminated by this filter were retained as potentially downregulated probe sets. The next filter was designed to eliminate probe sets that could not meet a minimal criterion for upregulation. Starting with the 3P filtered list, we eliminated all probe sets that did not have average values $>1.5$ in at least two conditions (time points). Those that were not eliminated by this filter were retained as potentially upregulated probe sets. However, there were a small number of probe sets that were not eliminated by either filter. Using a Venn diagram, we removed these from both lists and created a list of probe sets with potential complex regulation. Figure 5 shows gene trees of the potential downregulated probe sets $(1,466)$, upregulated probe sets $(1,412)$, and a group of probe sets $(60)$ that met both criteria.

The last filter addressed the quality of the data. For this "quality control" filter, we eliminated probe sets that did not meet two conditions. The first condition focused on the control chips. As indicated above, our initial operation was to divide the value of each individual probe set on each chip by the mean of the values for that probe set on the four control chips. Therefore, the quality of the control data for each probe set is of particular importance in defining regulation by the drug. This filter eliminated probe sets whose control values exhibited CVs $>50 \%$. The CV\% of the entire data set was $44.8 \%$, and that of the controls was $44.6 \%$. The $50 \%$ cutoff was chosen to approximate the quality of the entire data set. The second condition focused on the remaining 10 time points. This filter also eliminated probe sets whose $\mathrm{CV}$ for more than 5 of the remaining 10 time points exceeded $50 \%$. Following the application of this filter, 2,316 probe sets remained for consideration. Of the 2,316, 1,226 were in the upregulated list, 1,065 were in the downregulated list, and 25 were in the list that met both criteria. Figure 6 shows a gene tree of all 2,316 remaining probe sets. The three lists are provided as Supplemental Material (supplemental data are available at the online version of this article).

\section{RESULTS}

Figure 7, top, presents concentrations of MPL in plasma of animals receiving chronic MPL administration through Alzet pumps. By $6 \mathrm{~h}$, MPL concentrations reach a stable steady state that is maintained throughout the 7-day infusion period. In contrast, single MPL doses (Fig.

Physiol Genomics. Author manuscript; available in PMC 2014 October 06. 
7, bottom) result in drug concentrations that dissipate in a biexponential fashion and fall below the level of detection by $7 \mathrm{~h}$. Analyses of MPL kinetics for both acute $(40,42)$ and chronic $(35,36)$ dosing have been described previously.

Data mining of gene arrays from the chronic MPL treatment series identified 2,316 MPLregulated probe sets on the R230A gene chips. Muscle samples from animals previously given single-dose MPL were collected in a time series that involved 16 time points over a 72-h period. RNAs from those muscles were applied to the older Affymetrix RU34A chip. A similar filtering scheme applied to that data set identified 653 probe sets as being regulated by MPL. Using Affymetrix homology tables and basic local alignment search tool (Blast) searches, we identified 241 probe sets of the 653 on the U34A chip that corresponded to 219 of the 2,316 identified probe sets on the U230A chip. Because both chips in some cases contain multiple probe sets for the same gene, and because there is a higher degree of probe set redundancy on the older U34A chip, the numbers of corresponding probe sets common to the two chips are not equal. Likewise, the number of genes actually represented in this common set is less than the number of probe sets.

We identified 196 genes that were regulated by MPL in response to both dosing regimens. Most likely, this list does not contain all genes regulated by MPL in skeletal muscle. A perusal of both data sets indicates that there were many probe sets that failed the quality control filter on one of the two chips and were thus eliminated. Nonetheless, these 196 genes have a very high degree of certainty of being regulated by MPL in the muscle. In addition, the two profiles taken together provide an important foundation for understanding the mechanisms underlying the drug's regulation of genes in the skeletal muscle.

\section{Diversity of response profiles}

When the individual probe set profiles from acute and chronic dosing are viewed together, it is clear that multiple patterns of changes in mRNA expression occur. For example, Figs. 810 show representative examples of diverse response profiles of enhanced mRNA expression. Figure 8 shows acute and chronic expression profiles of two genes, mitogenactivated protein kinase-14 (MapK14) and peroxisome proliferator-activated receptor- $\delta$ (PPAR $\delta$ ), where there is a transient enhanced expression with return to baseline following both treatments. These profiles are very similar to the expression of tyrosine aminotransferase (TAT) in liver, a well-established marker for the enhancement of gene expression by glucocorticoids. We have previously characterized TAT mRNA expression by Northern hybridization in livers taken from the same animals used in these acute and chronic studies $(35,40)$. Like TAT, both MapK14 and PPAR $\delta$ exhibit tolerance in that these genes almost recover to their baseline after a period of time despite the continuous presence of the drug (35). Tolerance is not unexpected in light of the well-documented phenomena of glucocorticoid-induced downregulation of its own receptor. It should be noted that in earlier reports, we showed that the glucocorticoid receptor (GR) is strongly downregulated in response to MPL in both muscle and livers from these same animals (34-36, 40, 41). Since GR mediates the effect of the drug, the large reduction in this effector molecule should greatly reduce the driving force for changes in gene expression. Therefore, for genes exhibiting enhanced expression mediated by GR binding to glucocorticoid response 
elements (GREs; primary regulation), it is expected that both acute and chronic dosing would produce an initial enhanced expression followed by a return to baseline despite the continuous presence of the drug.

However, not all profiles exhibiting enhanced expression show tolerance. For example, Fig. 9 shows the response profiles of two genes, glutamine synthetase (GS) and eukaryotic translation initiation factor-4E binding protein-1 (Eif4ebp1), following both acute and chronic treatment. In both cases, the acute profile is very similar to those of the genes discussed above, with a transient increase followed by a return to baseline. It should be noted that we have previously reported this same profile for GS expression in muscles following acute dosing, as measured by Northern hybridization (34). However, the response profile of both genes presented in Fig. 9 to chronic infusion is entirely different, in that enhanced expression remains for at least $96 \mathrm{~h}$.

The response profiles shown in Figs. 8 and 9 do not encompass the entire repertoire of acute and chronic response profiles. For example, Fig. 10 shows the response profiles of interleukin-6 receptor (IL6R1) and interferon-related developmental regulator-1 (Ifrd1). Again, with acute treatment, there is a sharp peak of enhanced expression followed by a return toward baseline. With chronic treatment, there is an initial enhanced expression that appears to initially decrease toward baseline, but this is followed by a second higher level of enhanced expression. These genes appear as if they are going to return toward baseline, but at some time after $24 \mathrm{~h}$ of infusion some additional enhancing influence seems to intervene.

Thus, when the response profiles to two different dosing regimens are viewed together, it is very clear that a single profile by itself is not adequate to group genes into clusters with common mechanisms of regulation. A diversity of response profiles also exists for genes whose expression is downregulated by MPL. Figure 11 shows the response profiles of Syndecan 2, core protein ( $\mathrm{Sdc} 2)$. Both the acute and chronic profiles show a transient downregulation and a return toward baseline but an inability to fully recapture the baseline. In contrast, both the chronic and acute profiles of $\mathrm{Na}-\mathrm{K}-\mathrm{Cl}$ co-transporter (Nkcc1), presented in Fig. 12, show a transient downregulation followed by a period of enhanced expression with an eventual return toward baseline. Another variation in the chronic expression profile of genes that show downregulation are those with little or no recovery, as can be seen in Fig. 13. Both myristoylated alanine-rich C-kinase substrate (Macs) and extracellular signalrelated kinase-3 (ERK3) show a transient downregulation in the acute profile. However, in the chronic profile, Macs shows almost no recovery of baseline throughout the infusion period, while ERK3 shows a very slow progression back toward baseline.

Some genes exhibited more complex patterns involving both enhanced and repressed regulation. As illustrated by myogenic factor-6 (Myf6), presented in Fig. 14, the acute profile showed an initial downregulation in the first $4 \mathrm{~h}$ after dosing followed by a secondary upregulation. The chronic profile exhibited only the secondary enhanced regulation. Most likely, the chronic profile was unable to capture the initial downregulation because the first time point was taken after $6 \mathrm{~h}$ of infusion. By necessity, this was the first time point in this study because steady-state plasma MPL was only obtained at $6 \mathrm{~h}$ after pump implantation. 
Together, the data presented in Figs. 8-14 illustrate that changes in gene expression in response to corticosteroids clearly involve multiple mechanisms.

\section{Gene groupings and expression profiles}

Of the total of 196 genes, $33 \%$ were downregulated, $43 \%$ were upregulated, and the remaining $24 \%$ were classified as complex following one or both dosing regimens. We conducted a literature search for all 196 genes primarily using the NCBI "search across databases" feature. On the basis of this information and domain knowledge, we grouped the 196 genes based primarily on function with two additional groups based on subcellular localization (mitochondrial and plasma membrane). These groups are presented in Tables 18 and list identifying criteria as well as temporal responses following both acute and chronic treatments. For each gene on the tables, we provide the probe set identification number for the gene on the 230A chip (chronic) and the U34A chip (acute). We also characterize the response of the gene to both dosing regimens as upregulated (up), downregulated (down), or complex (some combination of both). Although not perfect, these groupings together with the expression profiles provide insight into the global impact of corticosteroids on the skeletal muscle.

The most highly populated group is termed "transcription-translation" and contains 47 genes (Table 1). The majority of genes in this group are transcription factors, indicating that a major influence of corticosteroids is derived from their ability to alter the effect of other influences on transcription. Alteration in expression of a transcription factor would likely serve as a secondary biosignal altering the expression of other genes. Since alterations in the amount of messages only have an impact once they are reflected as changes in protein, the consequence of these changes should be delayed in time. Such a time delay may therefore explain at least some of the genes that express complex higher-order regulation. Of particular interest in this group, because of their relationship to the control of muscle gene expression (11), is the downregulation in both response profiles of myogenin and myogenic factor D (MyoD), as well as the more complex response of myogenic factor-6 (Fig. 14), which shows upregulation in the acute profile and transient downregulation followed by sustained upregulation in the chronic profile.

A second highly populated group is termed "signaling," with 37 members (Table 2). Thirteen genes in this group show upregulation in both the acute and chronic profiles and 15 show downregulation in both acute and chronic profiles. As might be expected, this group is dominated by kinases and phosphatases. We have also included in this group several membrane receptors that could have been included in the "membrane" group but were included in this group because of their involvement in signaling. As with transcription factors, alterations in the expression of signaling proteins may act as secondary biosignals that modulate the expression of other genes. Obviously missing in this group is myostatin, which is important to the maintenance of muscle mass $(22,24)$. Although this gene was retained in the filtering of the acute data set, it was eliminated by the quality control filter in the chronic data set because the CV of the controls was $80 \%$. Because of the potential importance of this gene to muscle atrophy, Fig. 15 provides the gene array data of acute and chronic profiles for myostatin. Also presented in Fig. 15 is myostatin message measured by

Physiol Genomics. Author manuscript; available in PMC 2014 October 06. 
quantitative RT-PCR after chronic treatment, confirming the profile obtained by arrays. In both chronic and acute profiles, there is a strong upregulation followed by a rather shallow but sustained downregulation.

The group termed "plasma membrane" contains 23 genes (Table 3). Because the surface membrane of a cell mediates its interaction with the external environment, this group by necessity is difficult to distinguish from the signaling group. The "immune-related" group contains 18 genes (Table 4). Some of these genes, such as interleukin-6 receptor and the interferon- $\gamma$ receptor, could be included in the signaling or plasma membrane groups. However, because the pharmacological use of corticosteroids is to modulate immune/ inflammatory processes, we chose to list these separately. The next most populated group is best characterized as "vascular" and contains 16 genes (Table 5). This group contains genes such as angiotensin-converting enzyme, which influences vascular tone (31), as well as genes such as vascular endothelial growth factor, which influences angiogenesis (27). The next group contains 12 genes related to "protein and amino acid metabolism" (Table 6). Two genes in this list are of particular interest because of their potential involvement in the muscle atrophy caused by corticosteroids: GS and ring finger protein-28 (MURF1) (13). As can be seen in Fig. 9, following the acute dose, there is a rather rapid rise in GS expression followed by a relatively slow decline. However, the chronic profile is rather unusual in that the mRNA remains elevated for an extended period of time and only declines after $96 \mathrm{~h}$. Figure 16 shows the profiles for MURF1. Although the acute profiles for GS and MURF1 are quite similar, the chronic profiles are somewhat different. Following chronic infusion, MURF1 reaches an early peak, remains high for $48 \mathrm{~h}$, and then declines quite slowly over the remainder of the 168-h infusion period. The next group of genes, containing 10 members, is termed "nuclear-encoded mitochondrial genes" (Table 7). The dominant trend in these genes is a decrease which may reflect the almost $30 \%$ loss in mass of the gastrocnemius muscle (36). Table 8 contains the remaining 34 genes and is classified as "other." Table 8 contains genes involved in various processes. For example, glutathione peroxidase-3 and microsomal glutathione $S$-transferase-2 are associated with oxidative stress. Table 8 also contains several genes involved in small molecule metabolism, such as sulfotransferase-1a1, cytochrome $P-4504 \mathrm{~b} 1$, cytosolic cysteine dioxygenase-1, flavincontaining monooxygenase-1, and $S$-adenosylmethionine decarboxylase-1, all of which are upregulated on both profiles (19). Finally, Table 8 contains four expressed sequence tags that could not be identified by Blast searches.

\section{DISCUSSION}

This report describes the mining of a microarray data set obtained from the analysis of skeletal muscles from a population of adrenalectomized animals given a chronic infusion of MPL for up to $1 \mathrm{wk}$. Muscle RNA from 4 control animals and 4 animals killed at each of 10 time points over a 168 -h period was applied to individual Affymetrix R230A chips. The data set was mined using a filtering approach similar to the one applied to data sets developed from skeletal muscles, livers, and kidneys of animals treated with a single bolus dose of MPL, where animals were killed at 16 time points following dosing and compared with untreated controls $(4,6,7)$. This filtration approach does not select for probes but rather 
eliminates probe sets that do not meet explicit requirements. Those probe sets not eliminated are retained for analysis.

The filtration yielded a remainder of 2,316 probe sets for further consideration. These probe sets were compared with 653 probe sets that remained following filtering of the data set obtained following the single dose of MPL (6). This comparison yielded 241 probe sets in the acute-dosing data set that corresponded to 219 probe sets in the chronic infusion data set. The results identified 196 different genes regulated by both dosing regimens. Because the filtering process is quite stringent, these 196 genes most likely do not include all genes regulated by MPL in the tissue. However, they do provide a basis for evaluating the global effects of corticosteroids on skeletal muscle. It is probable that, had we employed one of the other presently available probe set algorithms, we would have obtained a broader selection of probe sets. All of these algorithms have strengths and weaknesses, with none being optimal for all purposes, especially time series data. For example, GC-RMA shows good performance in organisms with few confounding variables (e.g., yeast) but results in many false positives in rats (38). Our initial analysis was quite stringent and employed no single algorithm. However, given the public availability of our data sets and the active evolution of algorithms for mining and clustering gene array time series data sets, we expect that these data sets will be revisited in the future as new tools become available. Of particular potential for these two data sets is the evolution of algorithms for biclustering (15).

The objective of obtaining two time series profiles for each gene is to identify genes with common mechanisms of regulation. The hypothesis is that if two or more genes have common mechanisms of regulation, then they should have the same temporal profile in response to all dosing regimens. There are available a variety of clustering methods designed to group genes based on their profiles $(8,9)$. However, at present, no analytic method is available to cluster using two time series profiles, viz., "biclustering." Our filtering approach crudely identifies genes that meet minimal criteria for "up-" and "down"regulation based on deviation from baseline. In both the acute and chronic data sets, some profiles met both criteria, suggesting complex regulation. We visually inspected all profiles (241 acute and 219 chronic) and categorized each based on up- or down-deviation from baseline. In many cases, because of probe set redundancy, more than one profile was available for acute, chronic, or both. These instances are noted on the tables. With this information, we proceeded to evaluate acute and chronic profiles together. Solely on the basis of this crude classification, we identified three basic patterns. For $>75 \%$ of the genes, the profile was either up or down in both the acute and chronic profiles. In the remaining cases, the profiles were complex, involving both up- and downregulation. However, closer analysis, as presented in Figs. 8-16, illustrates that, even with the consideration of the $<75 \%$ of the genes where the profiles were either both up or both down, there still remains considerable diversity in mRNA expression patterns and therefore presumably in mechanisms of regulation.

The generally accepted mechanism for most glucocorticoid effects involves binding of free steroid to a cytoplasmically localized receptor, translocation of ligand-bound receptor to the nucleus, binding of a ligand receptor dimer to a specific DNA site (GRE), and modulation of the amount of selective mRNAs (42). Although some effects on mRNA stability have been 
noted, a common mechanism involves increasing or decreasing the rate of transcription of particular genes. In studies of the enzyme TAT in liver involving both a repeated dosing and a chronic-dosing paradigm, we described the phenomena of steroid tolerance $(35,36,41)$. In those and other reports, we demonstrated that MPL treatment caused a rather long-lived downregulation of the glucocorticoid receptor (mRNA and protein) in both skeletal muscle and liver. In addition, when a second dose was administered before full recovery of receptor, the enhanced expression of TAT (mRNA and protein) was reduced in proportion to the reduced concentration of receptor (41). In subsequent studies, we found that chronic infusion of MPL caused a sustained downregulation of the receptor (mRNA and protein) and that the expression of TAT (mRNA and protein) returned back toward the baseline in the continuous presence of the drug $(35,36)$. If the glucocorticoid receptor alone mediates the effect of the drug, then this is a rational result. Figures 8 and 15 show that the acute and chronic profiles of MAPK14, PPAR $\delta$, and myostatin come close to approximating a chronic profile of tolerance. Similarly, Fig. 11 to some degree approximates this result for downregulation. However, throughout the course of MPL infusion, the muscles continuously atrophied (36). Thus the response of the muscles to the continuous infusion suggests that those genes involved in effecting the atrophy should not show tolerance. It has been suggested that upregulation of myostatin may be involved in chronic muscle atrophy . However, the fact that myostatin only shows a transient upregulation followed by downregulation suggests that myostatin may not be involved in chronic atrophy caused by infusion of MPL. In contrast, the more sustained upregulation of GS, MURF1, and Eif4eBP supports their involvement in chronic atrophy. Similarly, the sustained downregulation of Macs and ERK3 supports their involvement in the chronic and continuous effects of corticosteroids on skeletal muscle, such as insulin resistance and atrophy (5).

However, the major question posed by a perusal of the profiles that do not show tolerance is as follows. How can the drug continue to have sustained strong effects when the receptor is greatly diminished to the point of almost being gone? One rational possibility is that our concept of the structure and function of the GRE is entirely too simplistic. If multiple GREs with greatly different affinities for the drug receptor complex exist, then the type of result seen for genes that show more sustained up- or downregulation could be explained. An additional complexity might operate if different isoforms of GR, as suggested by the work of Lu and Cidlowski (32), are interacting with multiple GREs. Another possibility is that our concept of only GR mediating corticosteroid effects may be simplistic, and some other "receptor" or interactions with other transcription factors are involved. This possibility is suggested by the chronic profile for IL6R and Ifrd-1 seen in Fig. 10. Initially, it appears as if both of these genes are going to respond with patterns similar to profiles for Mapk14 and PPAR $\dagger$ and return to baseline, but the expression is enhanced again to even higher levels. An alternative but related possible mechanism that could result in similar patterns of expression is the regulation of a secondary biosignal by glucocorticoids that in turn further modulates the expression of a glucocorticoid-regulated gene. Obvious candidates for potential biosignals would, of course, be other known transcription factors. As illustrated in Table 1, a large number of transcription factors are themselves modulated by MPL. In any case, the results clearly demonstrate that at present, there is much we do not know about how corticosteroids influence gene expression. 
We attempted to sort the 196 genes into groups based on a domain knowledge ontology.

Table 1 demonstrates the broad impact these drugs have on transcription and translation. Of particular interest is the downregulation in both the acute and chronic profiles of myogenin and MyoD. These are two of four basic-helix-loop-helix transcription factors that are important for expressing muscle phenotype $(11,22)$. The upregulation of Id1 in both profiles is consistent with a reduced influence of these muscle regulatory factors, since Id 1 has been shown to bind to and inhibit these basic-helix-loop-helix transcription factors in skeletal muscle (47). Unexpectedly, a third one of the four, Myf6, shows a transient downregulation followed by upregulation in the acute profile and a delayed upregulation in the chronic profile (Fig. 14). It should be noted that the fourth member of this group of transcription factors, Myf5, is not represented by a probe set of the Affymetrix chips. The upregulation of both PPARa and PPAR $\delta$ is also quite relevant to the condition of the metabolic syndrome caused by corticosteroids. Both transcription factors are important for regulating fatty acid oxidation in skeletal muscle and may relate to corticosteroid-induced insulin resistance (5, 21,39 ).

In interpreting these results, it is important to keep in mind that skeletal muscles contain satellite cells in addition to multinucleated fibers. The downregulation of Meox2, a gene that appears to be upstream of the expression of both MyoD and myogenin (33), suggests the possibility that corticosteroids are suppressing the expression of muscle phenotypes in the satellite cell population.

Although the signaling table is dominated by kinases and phosphatases, one unexpected result is the downregulation of IGFBP5 in both profiles. It has been suggested that the upregulation of IGFBP5 may play a role in muscle atrophy by sequestering IGF-I (26). Interestingly, IGFBP3 is upregulated in both profiles. However, the fact that corticosteroids down-regulate the expression of IGF-I may make changes in expression patterns of the binding proteins much less relevant to corticosteroid-induced atrophy (44). It has also been suggested that IL6 is associated with muscle atrophy conditions (44). The sustained upregulation of the IL6 receptor in both profiles is consistent with this possibility. Similarly, the sustained upregulation of Eif4ebp1 is consistent with corticosteroids inhibiting protein synthesis, while the upregulation of both MURF1 and GS is consistent with increased protein degradation $(13,29,34)$. Previously, we presented data supporting the hypothesis that both denervation and disuse atrophy are caused by a muscle becoming hypersensitive to circulating glucocorticoids $(1-3,16-18)$. In the intervening years, aspects of this hypothesis have been examined by others $(24,25,43)$. With the single exception that IGFBP5 is upregulated following hindlimb suspension, whereas this gene is clearly downregulated by corticosteroids, the initial hypothesis still seems reasonable.

The use of a rich time series such as this for examining patterns of changes in gene expression can be more informative than single time point studies for several reasons. The data presented here illustrate that, to simply categorize a gene as upor downregulated is too simplistic, as multiple different patterns of up- and downregulation occur following corticosteroid treatment. Characterization of the magnitude of expression change using single time point studies can also be misleading, since the magnitude of change will be dependent on the particular time point examined. In fact, with complex biphasic regulation, 
a change in expression may be categorized as up at one point in the time series but not changed or down at a different time point. For these reasons, we did not attempt to list magnitude of change in Tables 1-8.

Finally, the two data sets together illustrate the need for data mining tools that are capable of the coincidental analysis of more than one temporal profile. Our approach to filtering in many respects was too stringent. Not only was the upregulated myostatin marginally eliminated in one of the two filtered data sets, but also, other clearly relevant genes such as the downregulated insulin receptor substrate-1 (IRS-1) were eliminated by filtering in one of the two data sets. We anticipate that, as additional tools become available, these data sets will be revisited many times.

All data sets described in this and related cited publications are available online in GEO. It is our intent to make these data sets, as well as our related data sets on other tissues taken from these same animals, widely available to other researchers. For this reason, all data are also available online at the PEPR site (http://pepr.cnmcresearch.org) developed and maintained by the Children's National Medical Center, Microarray Research Center (14). These data are in a user friendly format, where individual temporal profiles are searchable and all data can be obtained and used without requirement for any additional specialized software.

\section{Supplementary Material}

Refer to Web version on PubMed Central for supplementary material.

\section{Acknowledgments}

GRANTS

This work was supported by National Institutes of Health (NIH; Bethesda, MD) Grants GM-24211 and GM-67650 and by a grant from the National Aeronautics and Space Administration. This data set was developed under the auspices of a grant from the National Heart, Lung, and Blood Institute/NIH Programs in Genomic Applications (HL-66614).

\section{REFERENCES}

1. Almon RR, DuBois DC. Adrenalectomy eliminates both fiber-type differences and starvation effects on denervated muscle. Am J Physiol Endocrinol Metab. 1988; 255:E850-E856.

2. Almon RR, DuBois DC. Are there conditions in which adrenalectomy impedes the atrophying effects of denervation? Physiologist. 1985; 28:S69-S70. [PubMed: 3834490]

3. Almon RR, DuBois DC. Fiber-type discrimination in disuse and glucocorticoid-induced atrophy. Med Sci Sports Exerc. 1990; 22:304-311. [PubMed: 2199752]

4. Almon RR, DuBois DC, Jin JY, Jusko WJ. Pharmacogenomic responses of rat liver to methylprednisolone: an approach to mining a rich microarray time series. AAPS J. 2005; 7:E156E194. [PubMed: 16146338]

5. Almon RR, DuBois DC, Jin JY, Jusko WJ. Temporal profiling of the transcriptional basis for the development of corticosteroid-induced insulin resistance in rat muscle. J Endocrinol. 2005; 184:219-232. [PubMed: 15642798]

6. Almon RR, DuBois DC, Piel WH, Jusko WJ. The genomic response of skeletal muscle to methylprednisolone using microarrays: tailoring data mining to the structure of the pharmacogenomic time series. Pharmacogenomics. 2004; 5:525-552. [PubMed: 15212590] 
7. Almon RR, Lai W, DuBois DC, Jusko WJ. Corticosteroid-regulated genes in rat kidney: mining time series array data. Am J Physiol Endocrinol Metab. 2005; 289:E870-E882. [PubMed: 15985454]

8. Androulakis, IP.; Vitolo, J.; Roth, CM. Proc Foundations Systems Biol Engineering. Santa Barbara, CA: 2005. Selecting maximally informative genes to enable temporal expression profiling analysis..

9. Arai K, Lee K, Berthiaume F, Tompkins RG, Yarmush ML. Intrahepatic amino acid and glucose metabolism in a d-galactosamine-induced rat liver failure model. Hepatology. 2001; 34:360-371. [PubMed: 11481621]

10. Bao S, Kennedy A, Wojciechowski B, Wallace P, Ganaway E, Garvey WT. Expression of mRNAs encoding uncoupling proteins in human skeletal muscle: effects of obesity and diabetes. Diabetes. 1998; 47:1935-1940. [PubMed: 9836527]

11. Bergstrom DA, Tapscott SJ. Molecular distinction between specification and differentiation in the myogenic basic helix-loop-helix transcription factor family. Mol Cell Biol. 2001; 21:2404-2412. [PubMed: 11259589]

12. Bialis M, Routledge P. Adverse effects of corticosteroids. Adverse Drug React Toxicol Rev. 1998; 17:227-235. [PubMed: 10196628]

13. Bodine SC, Latres E, Baumhueter S, Lai VK, Nunez L, Clarke BA, Poueymirou WT, Panaro FJ, Na E, Dharmarajan K, Pan ZQ, Valenzuela DM, Dechiara TM, Stitt TN, Yancopoulos GD, Glass DJ. Identification of ubiquitin ligases required for skeletal muscle atrophy. Science. 2001; 294:1704-1708. [PubMed: 11679633]

14. Chen J, Zhao P, Massaro D, Clerch L, Almon RR, DuBois DC, Jusko WJ, Hoffman E. The PEPR genechip data warehouse, and implementation of a dynamic time series query tool (sgqt) with graphical interface. Nucleic Acids Res. 2004; 32:D578-D581. [PubMed: 14681485]

15. Cheng Y, Church GM. Biclustering of expression data. Proc Int Conf Intell Syst Mol Biol. 2000; 8:93-103. [PubMed: 10977070]

16. DuBois DC, Almon RR. Disuse atrophy of skeletal muscle is associated with an increase in number of glucocorticoid receptors. Endocrinology. 1980; 107:1649-1651. [PubMed: 7428684]

17. DuBois DC, Almon RR. Glucocorticoid sites in skeletal muscle: adrenalectomy, maturation, fiber type, and sex. Am J Physiol Endocrinol Metab. 1984; 247:E118-E125.

18. DuBois DC, Almon RR. A possible role for glucocorticoids in denervation atrophy. Muscle Nerve. 1981; 4:370-373. [PubMed: 7290104]

19. Ehrhart J, Gluck M, Mieyal J, Zeevalk FD. Functional glutaredoxin (thioltransferase) activity in rat brain and liver mitochondria. Parkinsonism Rel Disord. 2002; 8:395-400.

20. Frauman A. An overview of the adverse reactions to adrenal corticosteroids. Adverse Drug React Toxicol Rev. 1996; 15:203-206. [PubMed: 9113247]

21. Guillet-Deniau I, Mieulet V, Le Lay S, Achouri Y, Carre D, Girard J, Foufelle F, Ferre P. Sterol regulatory element binding protein-1C expression and action in rat muscles: insulin-like effects on the control of glycolytic and lipogenic enzymes and UCP3 gene expression. Diabetes. 2002; 51:1722-1728. [PubMed: 12031958]

22. Guttridge DC. Signaling pathways weigh in on decisions to make or break skeletal muscle. Curr Opin Clin Nutr Metab Care. 2004; 7:443-450. [PubMed: 15192448]

23. Irizzary RA, Bolstad BM, Collin F, Cope LM, Hobbs B, Speed TP. Summaries of Affymetrix genechip probe level data. Nucleic Acids Res. 2003; 31:E15. [PubMed: 12582260]

24. Jackman RW, Kandarian SC. The molecular basis of skeletal muscle atrophy. Am J Physiol Cell Physiol. 2004; 287:C834-C843. [PubMed: 15355854]

25. Jagoe RT, Lecker SH, Gomes M, Goldberg AL. Patterns of gene expression in atrophying skeletal muscles: response to food deprivation. FASEB J. 2002; 16:1697-1712. [PubMed: 12409312]

26. Kamei Y, Miura S, Suzuki M, Kai Y, Mizukami J, Taniguchi T, Mochida K, Hata T, Matsuda J, Aburatani H, Nishino I, Ezaki O. Skeletal muscle FOXO1 (FKHR) transgenic mice have less skeletal muscle mass, down-regulated Type I (slow twitch/red muscle) fiber genes, and impaired glycemic control. J Biol Chem. 2004; 279:41114-41123. [PubMed: 15272020]

27. Kang DH, Johnson RJ. Vascular endothelial growth factor: a new player in the pathogenesis of renal fibrosis. Curr Opin Nephrol Hypertens. 2003; 12:43-49. [PubMed: 12496665] 
28. Kennedy B, Elayan H, Ziegler MG. Glucocorticoid hypertension and nonadrenal phenylethanolamine N-methyltransferase. Hypertension. 1993; 21:415-419. [PubMed: 8458643]

29. Lang CH, Frost RA. Endotoxin disrupts the leucine-signaling pathway involving phosphorylation of mTOR, 4E-BP1, and S6K1 in skeletal muscle. J Cell Physiol. 2005; 203:144-155. [PubMed: 15389631]

30. Locsey L, Asztalos L, Kincses Z, Gyorfi F, Berczi C. Dyslipidaemia and hyperlipidaemia following renal transplantation. Int Urol Nephrol. 1996; 28:419-430. [PubMed: 8899484]

31. Lodwick D, Kaiser MA, Harris J, Cumin F, Vincent M, Samani NJ. Analysis of the role of angiotensinogen in spontaneous hypertension. Hypertension. 1995; 25:1245-1251. [PubMed: 7768569]

32. Lu NZ, Cidlowski JA. Translational regulatory mechanisms generate N-terminal glucocorticoid receptor isoforms with unique transcriptional target genes. Mol Cell. 2005; 18:331-342. [PubMed: 15866175]

33. Mankoo BS, Collins NS, Ashby P, Grigorieva E, Pevny LH, Candia A, Wright CV, Rigby PW, Pachnis V. Mox 2 is a component of the genetic hierarchy controlling limb muscle development. Nature. 1999; 400:69-73. [PubMed: 10403250]

34. McKay LI, DuBois DC, Sun YN, Almon RR, Jusko WJ. Corticosteroid effects in skeletal muscle: gene induction/receptor autoregulation. Muscle Nerve. 1997; 20:1318-1320. [PubMed: 9324091]

35. Ramakrishnan R, DuBois DC, Almon RR, Pyszczynski NA, Jusko WJ. Fifth-generation model for corticosteroid pharmacodynamics: application to steady-state receptor down-regulation and enzyme induction patterns during seven-day continuous infusion of methylprednisolone in rats. J Pharmacokinet Pharmacodynam. 2002; 29:1-24.

36. Ramakrishnan R, DuBois DC, Almon RR, Pyszczynski NA, Jusko WJ. Pharmacodynamics and pharmacogenomics of methylprednisolone during 7-day infusions in rats. J Pharmacol Exp Ther. 2002; 300:245-256. [PubMed: 11752123]

37. Reynolds RM, Walker BR. Human insulin resistance: the role of glucocorticoids. Diabetes Obes Metab. 2003; 5:5-12. [PubMed: 12542720]

38. Seo J, Hoffman E. Probe set algorithims: is there a rational best bet? BMC Bioinformatics. 2006; 7:395. [PubMed: 16942624]

39. Staels B, Dallongeville J, Auwerx J, Schoonjans K, Leitersdorf E, Fruchart JC. Mechanism of action of fibrates on lipid and lipoprotein metabolism. Circulation. 1998; 98:2088-2093. [PubMed: 9808609]

40. Sun YN, DuBois DC, Almon RR, Jusko WJ. Fourth-generation model for corticosteroid pharmacodynamics: a model for methylprednisolone effects on receptor/gene-mediated glucocorticoid receptor down-regulation and tyrosine aminotransferase induction in rat liver. $\mathrm{J}$ Pharmacokinet Biopharm. 1998; 26:289-317. [PubMed: 10098101]

41. Sun YN, DuBois DC, Almon RR, Pyszczynski NA, Jusko WJ. Dose-dependence and repeateddose studies for receptor/gene-mediated pharmacodynamics of methylprednisolone on glucocorticoid receptor down-regulation and tyrosine aminotransferase induction in rat liver. $\mathrm{J}$ Pharmacokinet Biopharm. 1998; 26:619-648. [PubMed: 10485078]

42. Sun YN, McKay LI, DuBois DC, Jusko WJ, Almon RR. Pharmacokinetic/pharmacodynamic models for corticosteroid receptor down-regulation and glutamine synthetase induction in rat skeletal muscle by a receptor/gene-mediated mechanism. J Pharmacol Exp Ther. 1999; 288:720_ 728. [PubMed: 9918581]

43. Thissen JP. Towards an understanding of molecular mechanisms of muscle atrophy. [comment]. Curr Opin Clin Nutr Metab Care. 2005; 8:245-247. discussion 271-245. [PubMed: 15809525]

44. Tisdale MJ. Biochemical mechanisms of cellular catabolism. Curr Opin Clin Nutr Metab Care. 2002; 5:401-405. [PubMed: 12107376]

45. Wald JA, Farr RS. Abnormal liver-function tests associated with long-term systemic corticosteroid use in subjects with asthma. J Allergy Clin Immunol. 1991; 88:277-278. [PubMed: 1880328]

46. Wu Z, Irizzary RA. Preprocessing of oligonucleotide array data. Nat Biotechnol. 2004; 22:656658. [PubMed: 15175677] 
47. Wyzykowski JC, Winata TI, Mitin N, Taparowsky EJ, Konieczny SF. Identification of novel MyoD gene targets in proliferating myogenic stem cells. Mol Cell Biol. 2002; 22:6199-6208. [PubMed: 12167713] 


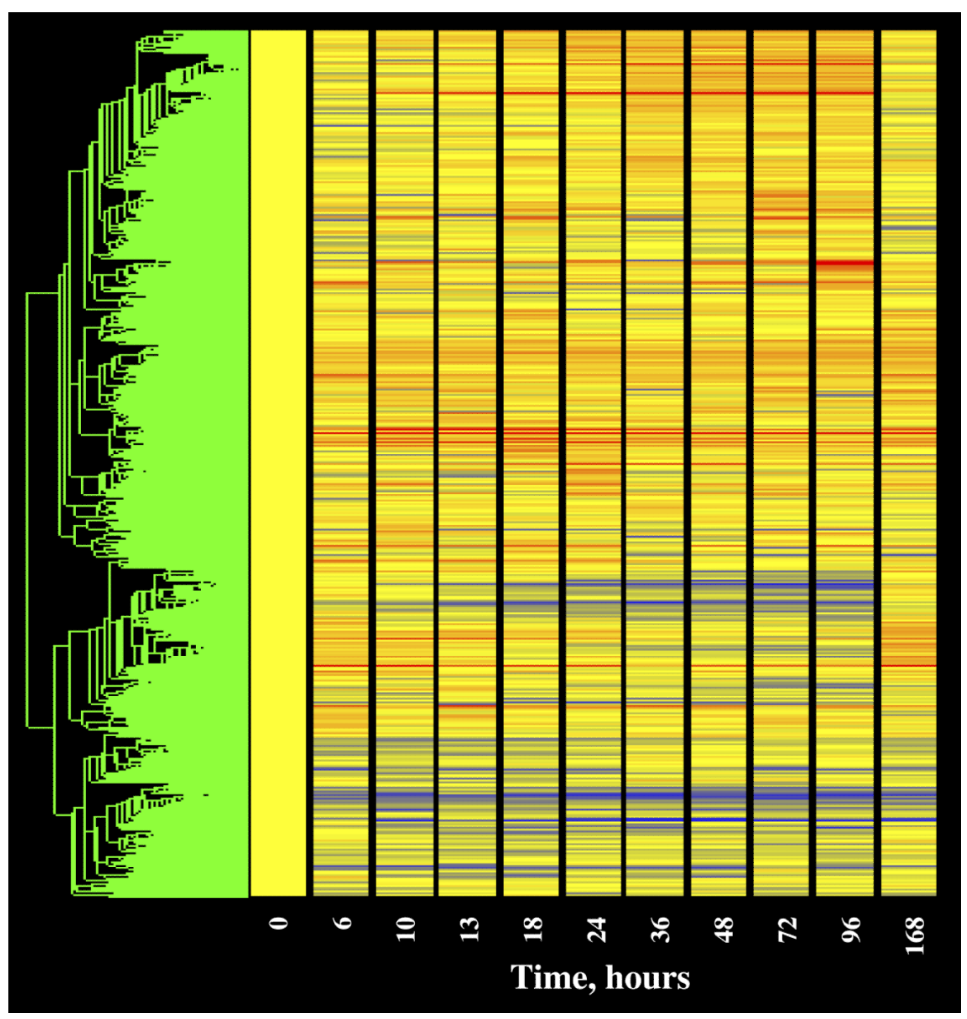

Fig. 1.

Gene tree representation of all probe sets $(15,967)$ on individual Affymetrix R230A gene chips hybridized to total RNA prepared from muscles taken from animals treated chronically with methylprednisolone (MPL; $0.3 \mathrm{mg} \bullet \mathrm{kg}^{-1} \bullet \mathrm{h}^{-1}$ ) for periods ranging from 6 to $168 \mathrm{~h}$. the values for each individual probe set at each time point were normalized to the mean value of that probe set for vehicle-treated controls. The $x$-axis represents the 11 time points, including vehicle-treated controls (nominal time 0 ). The $y$-axis presents the list of probe sets grouped by similarity using Pearson correlation. Yellow indicates no change from controls, red indicates probe sets with enhanced expression relative to controls, and blue indicates suppressed expression relative to controls. 

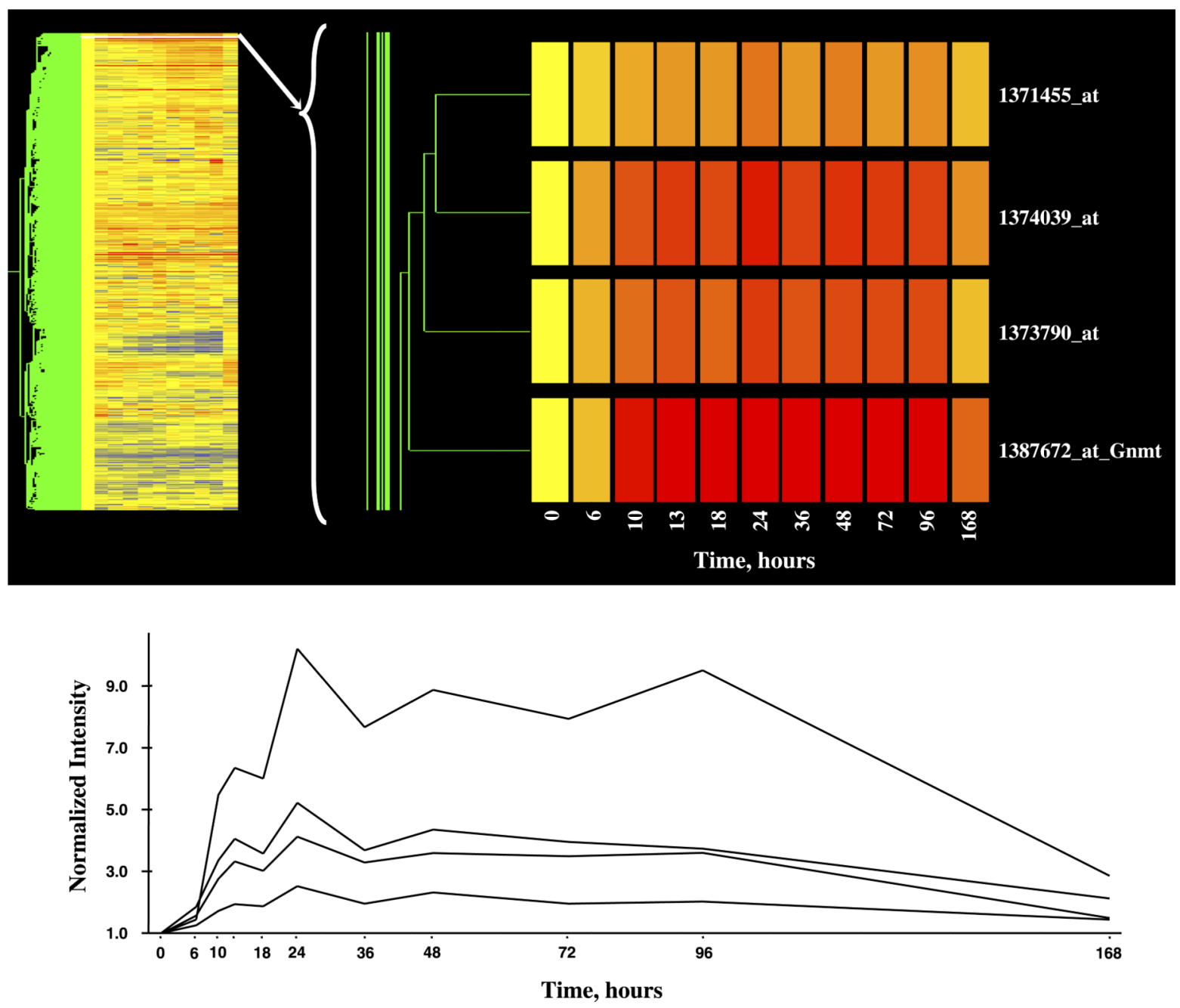

Fig. 2.

Top: a magnification of 4 probe sets selected from Fig. 1 that show apparent enhanced regulation by MPL. The linear plots for these probe sets are presented at bottom. 

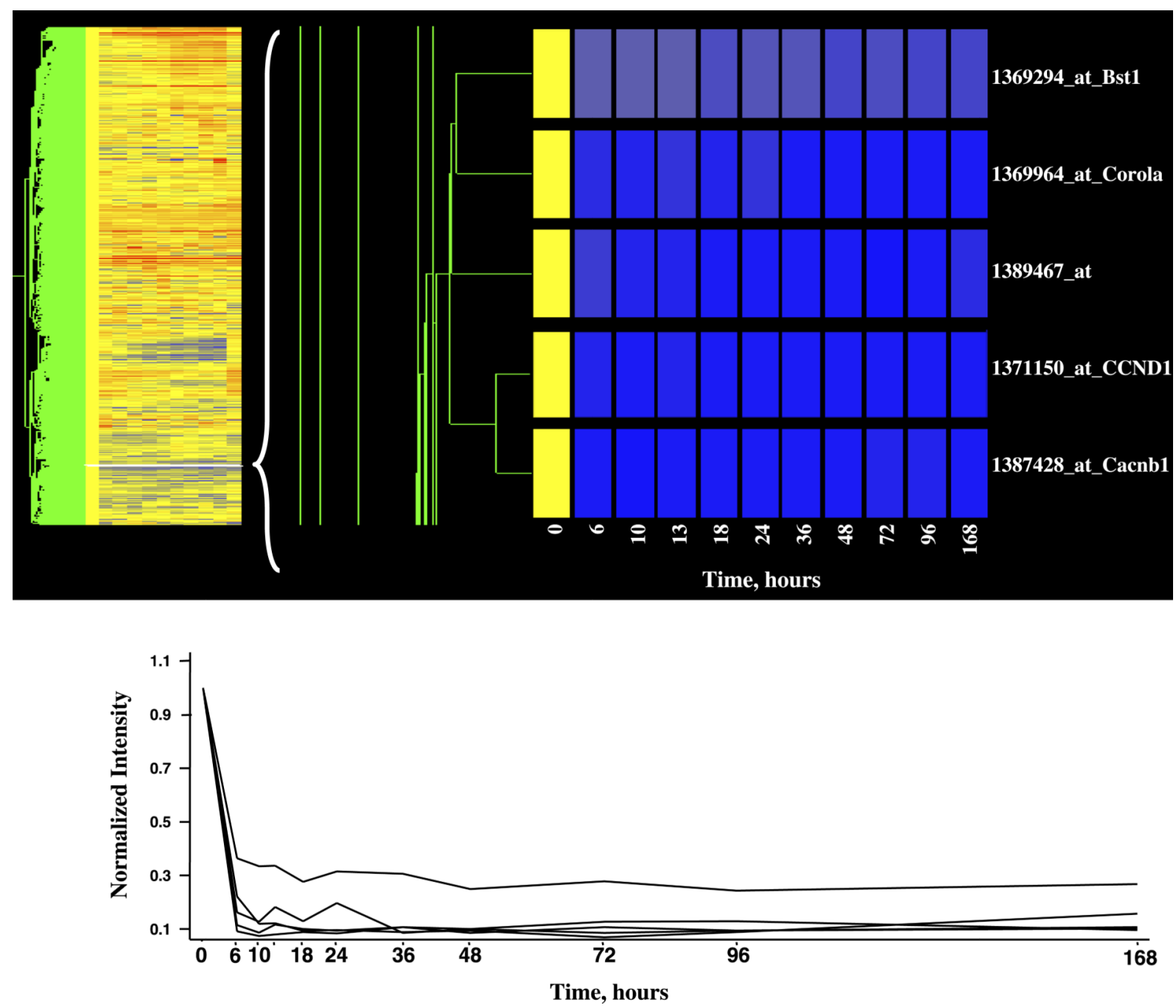

Fig. 3.

Top: a magnification of 5 probe sets selected from Fig. 1 that show apparent downregulation by MPL. The linear plots of all 5 probe sets are presented at bottom. 


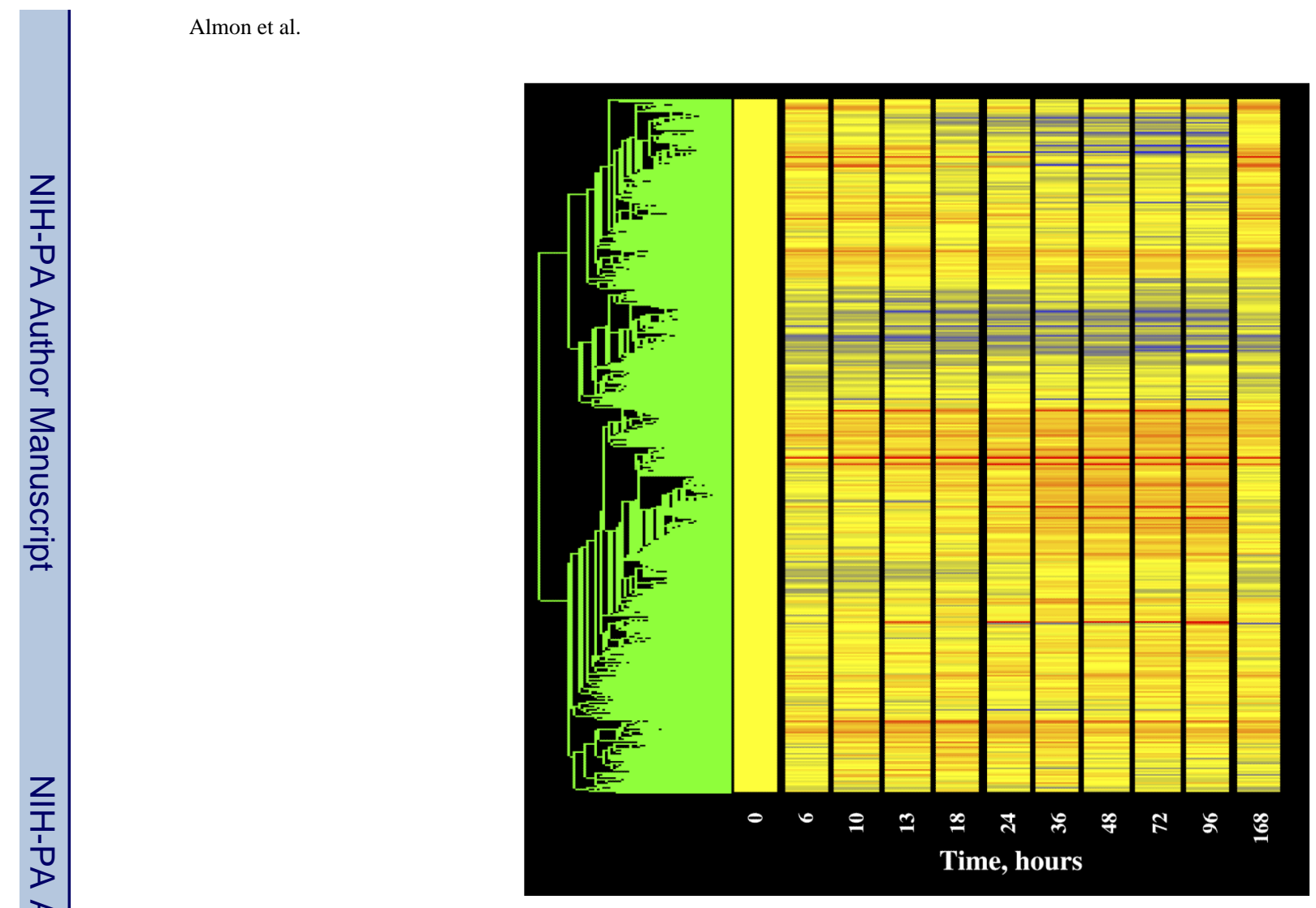

Fig. 4.

Gene tree representation of the 9,816 probe sets remaining after removing probe sets $(6,151)$ that did not give a call of present using MAS5.0 software in at least 3 of 44 chips in the data set. 


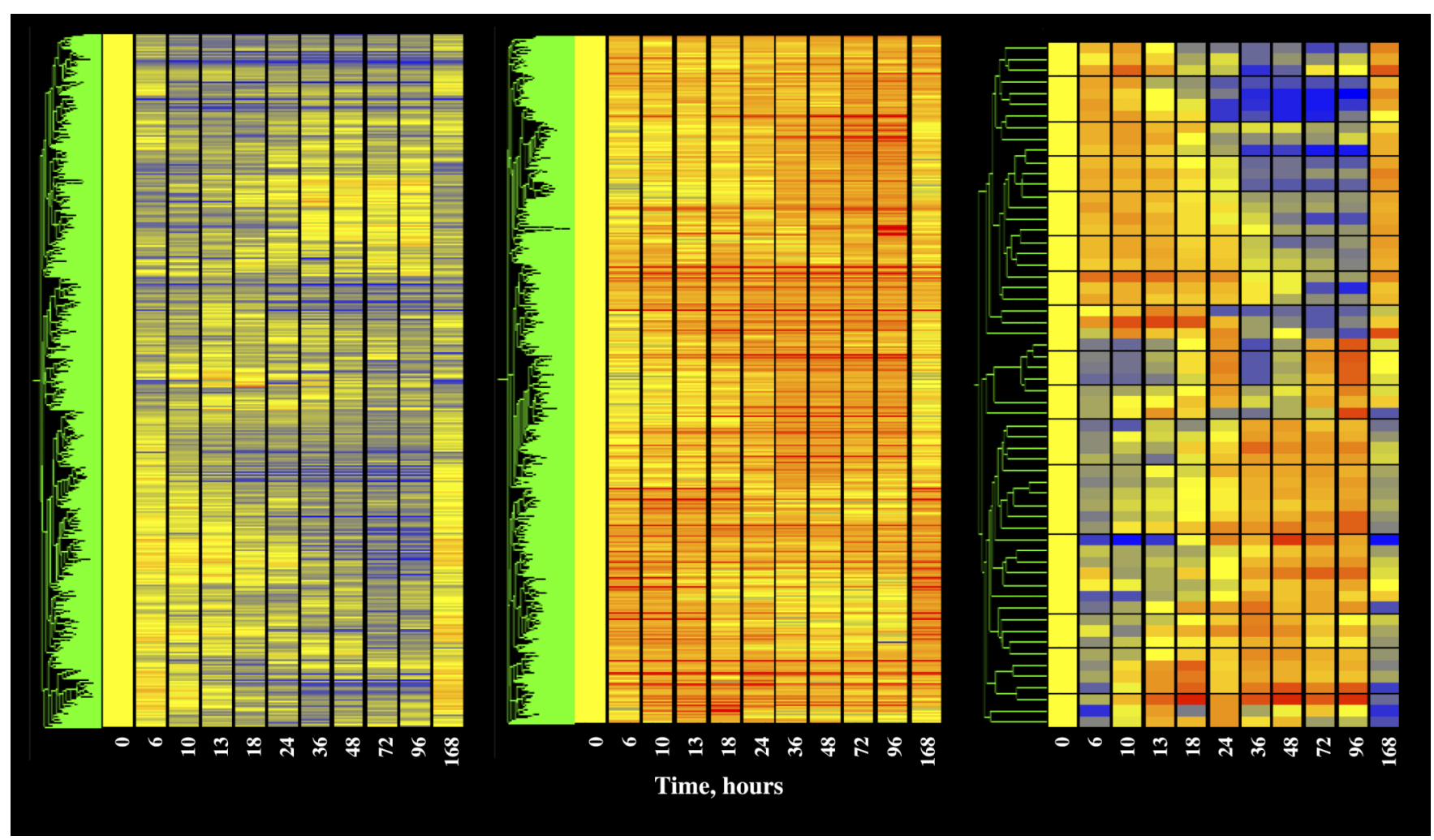

Fig. 5.

Gene trees of probe sets remaining after filtering for MPL regulation. Left: probe sets with potential downregulation $(1,466)$. Middle: those with potential upregulation $(1,412)$. Right: probe sets that met both criteria (60). 


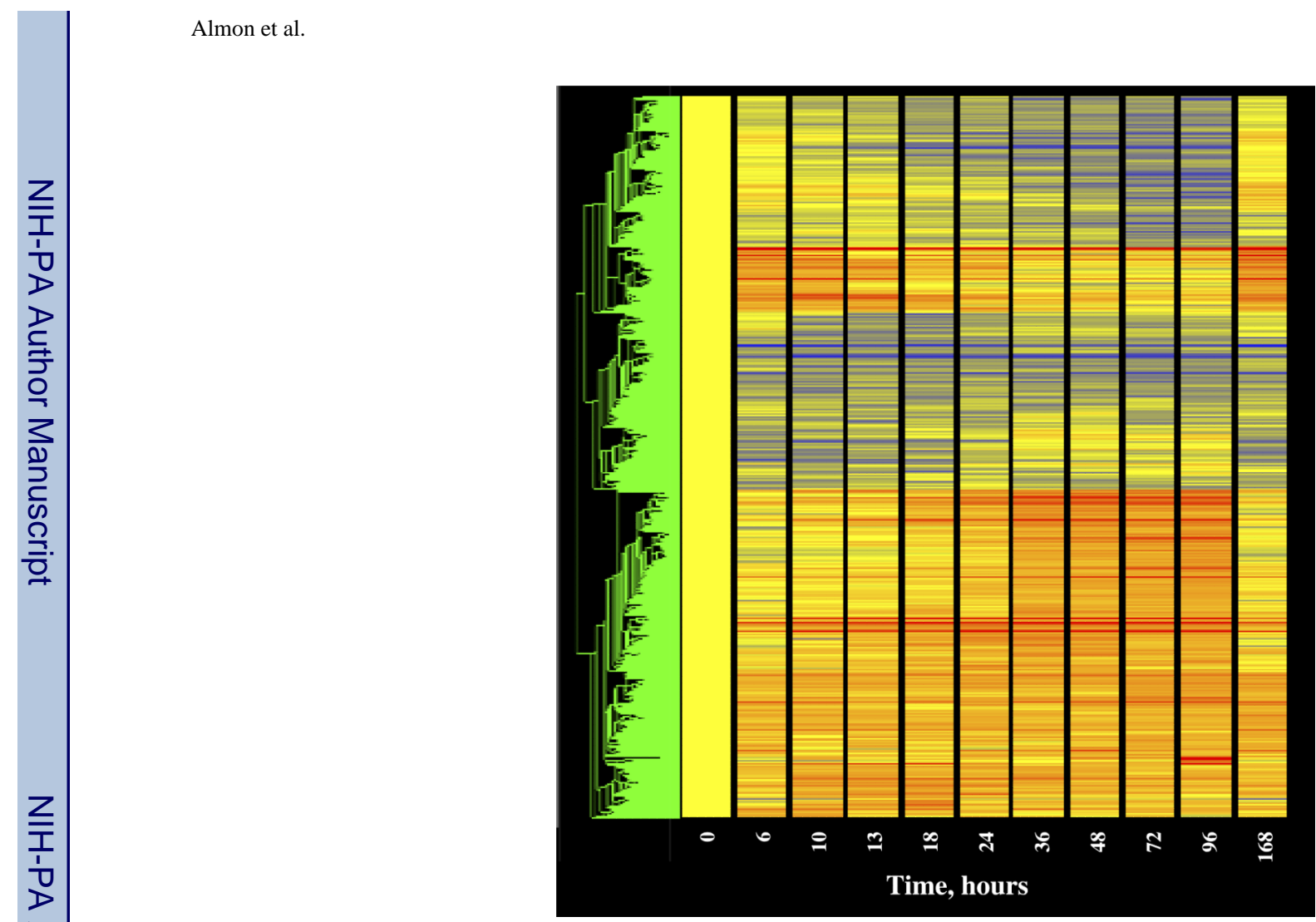

Fig. 6.

Gene tree representation of probe sets remaining $(2,316)$ after filtering the probe sets in Fig. 5 for high coefficients of variation. 

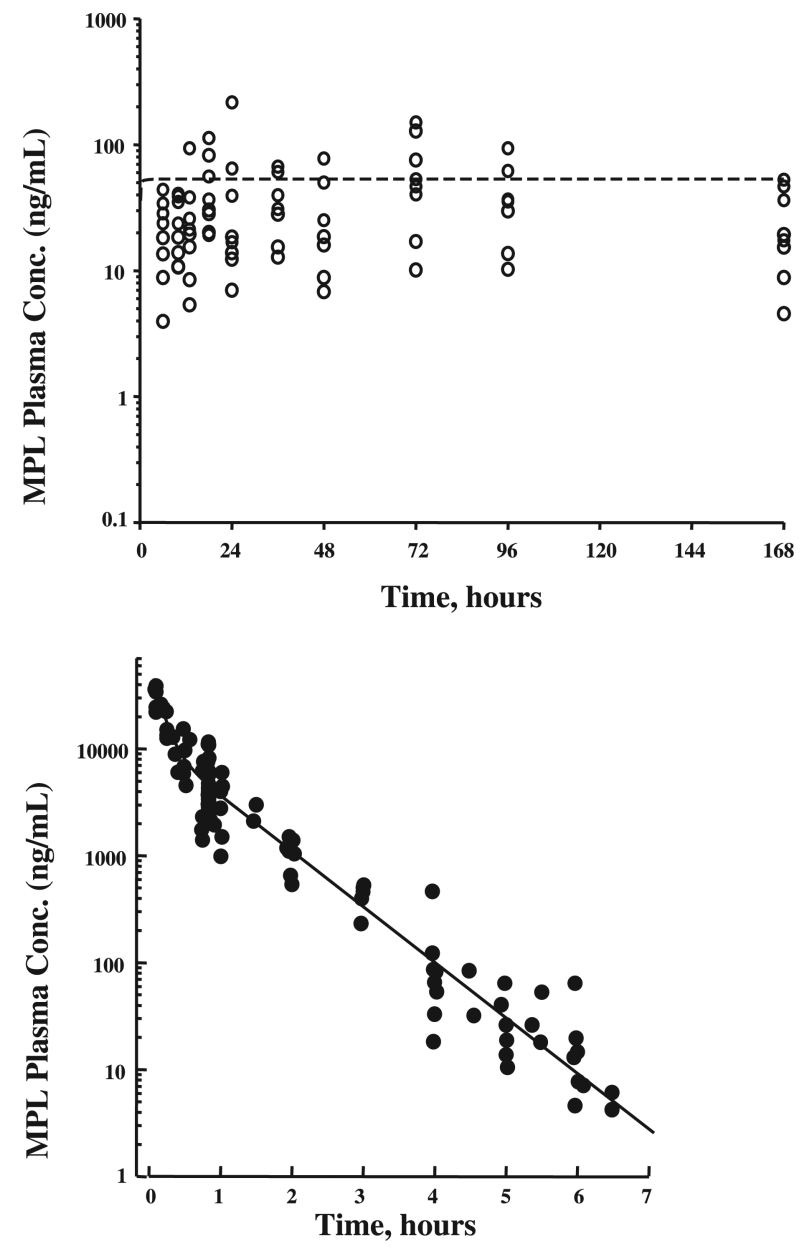

Fig. 7.

MPL concentrations in rat plasma following chronic (top) and acute (bottom) administration of drug. MPL concentrations were determined by normal-phase HPLC analysis of plasma samples obtained from individual animals. 
Mapk14

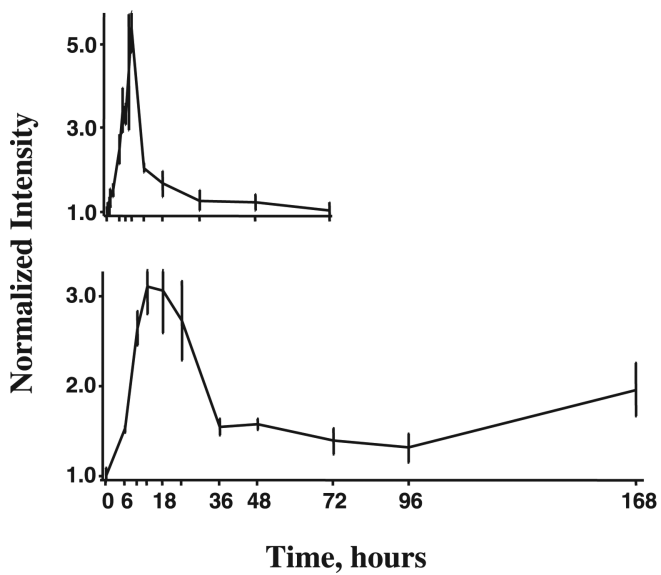

PPARdelta
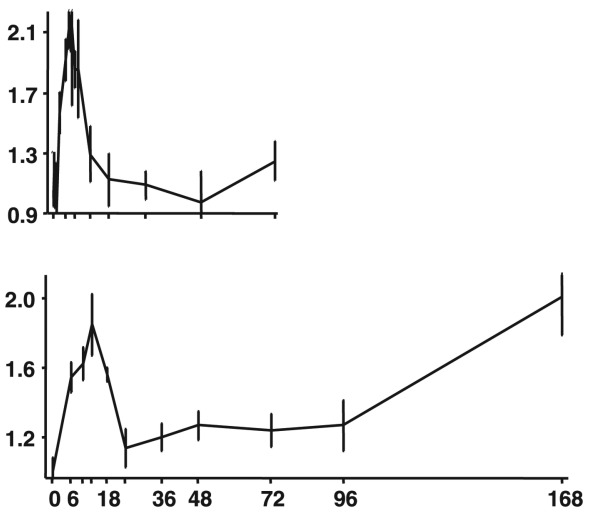

Time, hours

Fig 8.

Response profiles of 2 genes showing enhanced expression and tolerance with chronic dosing. Acute-dosing profiles are shown at top, and chronic-dosing profiles are shown at bottom. Mitogen-activated protein kinase-14 (MAPK14) expressions are shown at left and peroxisome proliferator-activated receptor- $\delta(\operatorname{PPAR} \delta)$ at right. 
GS

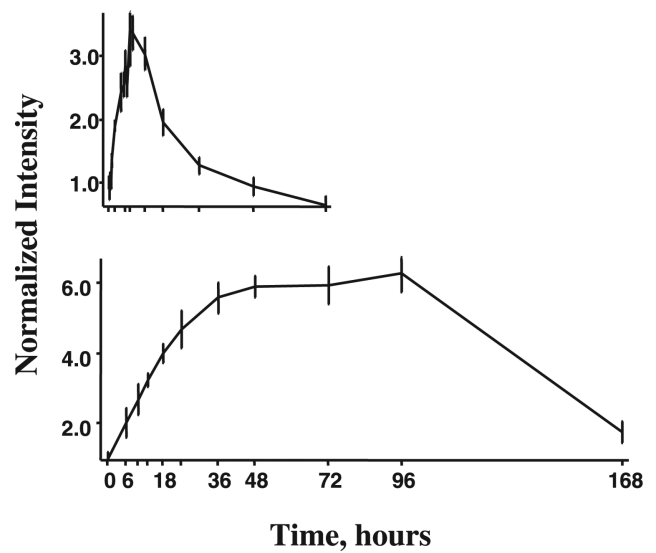

Eif4ebp1
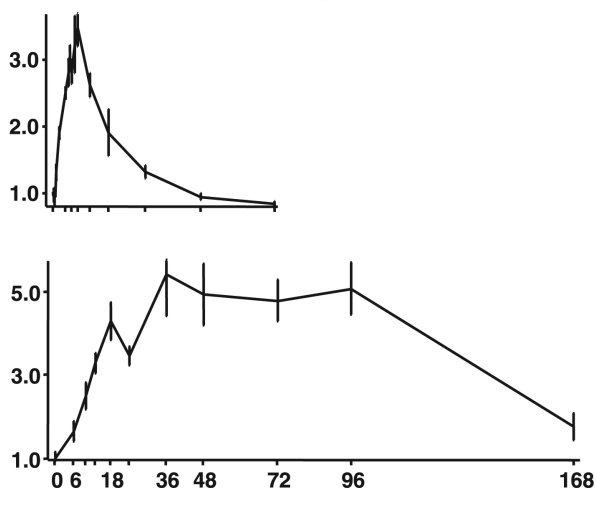

Time, hours

Fig. 9.

Response profiles of 2 genes showing enhanced expression but the absence of tolerance with chronic dosing. Acute-dosing profiles are shown at top, and chronic-dosing profiles are shown at bottom. Glutamine synthetase (GS) expressions are shown at left and eukaryotic translation initiation factor-4e binding protein-1 (Eif4ebp1) at right. 
IL6R1

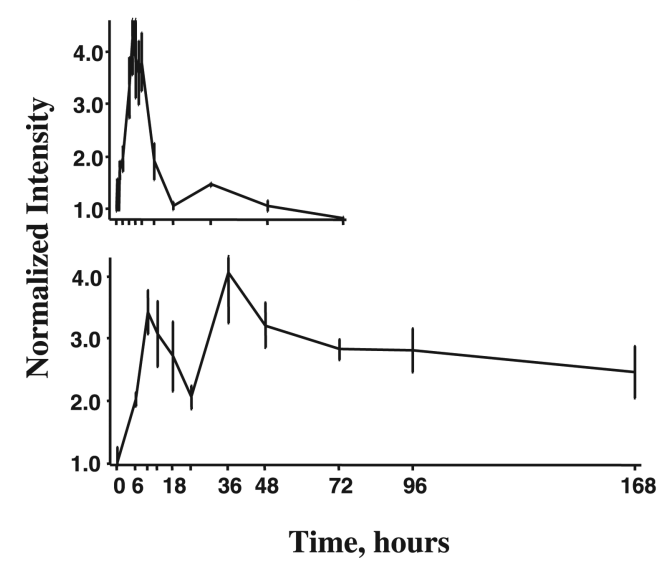

Ifrd1
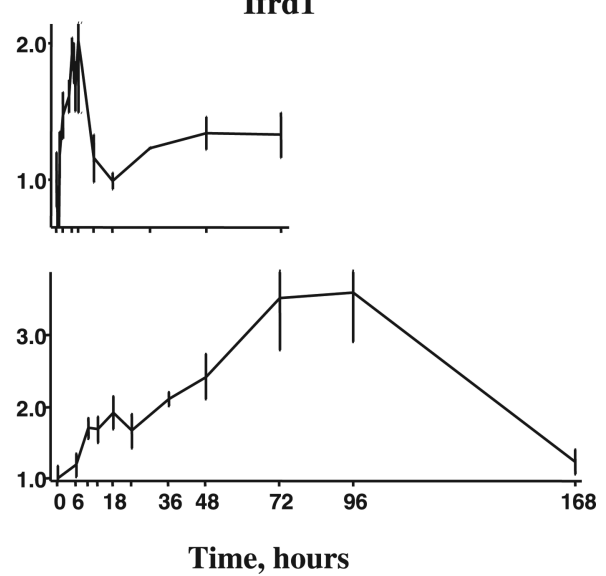

Fig. 10.

Response profiles of 2 genes showing both primary and secondary enhanced expression. Acute-dosing profiles are shown at top, and chronic-dosing profiles are shown at bottom. Interleukin-6 receptor (IL6R1) expressions are shown at left and interferon-related developmental regulator-1 (Ifrd1) at right. 
Sdc2

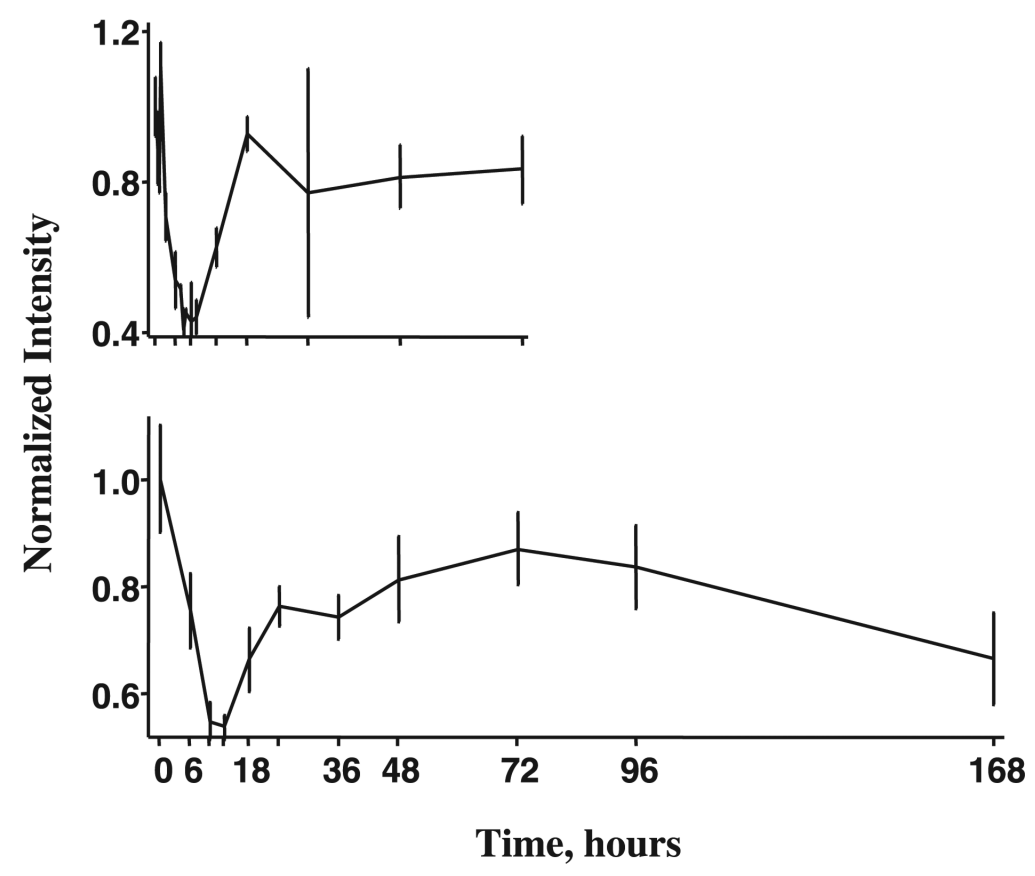

Fig. 11.

Response profiles of Syndecan 2 core protein (Sdc2), which shows decreased expression following MPL administration and only partial tolerance with chronic dosing. Acute-dosing profiles are shown at top and chronic-dosing profiles at bottom. 


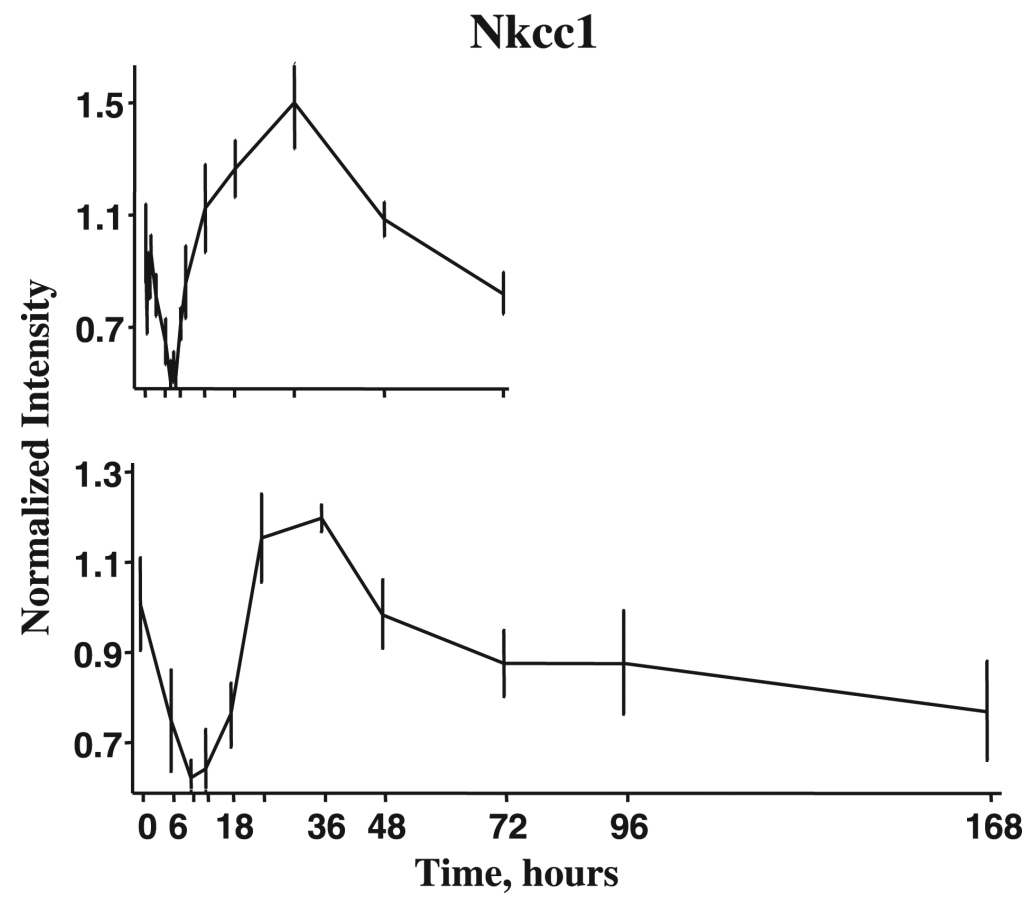

Fig. 12.

Response profiles of $\mathrm{Na}-\mathrm{K}-\mathrm{Cl}$ cotransporter ( $\mathrm{Nkcc1}$ ), which shows decreased expression following MPL administration and tolerance with chronic dosing. Acute-dosing profiles are shown at top and chronic-dosing profiles at bottom. 
Macs

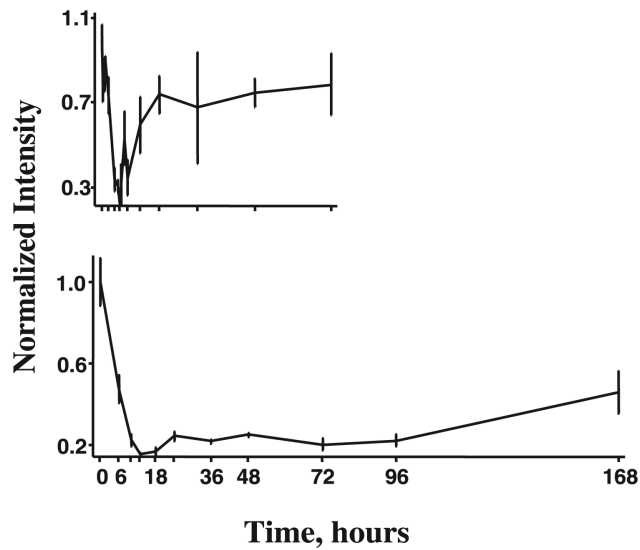

ERK3
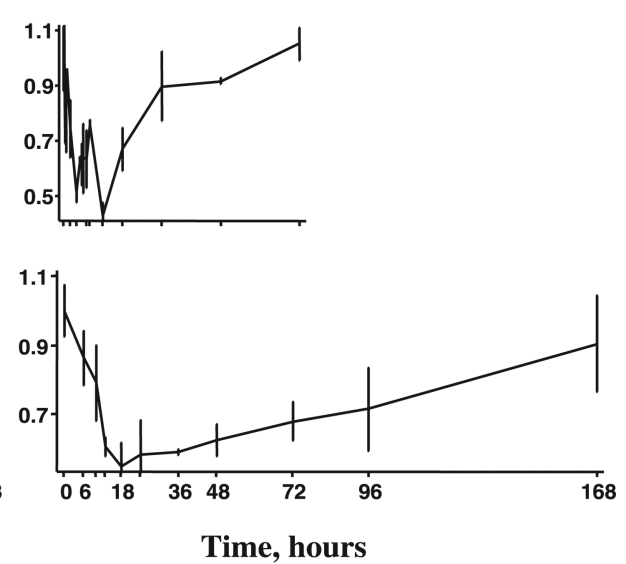

Fig. 13.

Response profiles of 2 genes showing decreased expression but absence of tolerance with chronic dosing. Acute-dosing profiles are shown at top, and chronic-dosing profiles are shown at bottom. Myristoylated alanine-rich C-kinase substrate (Macs) expression is shown at left and extracellular signal-related kinase-3 (ERK3) at right. 


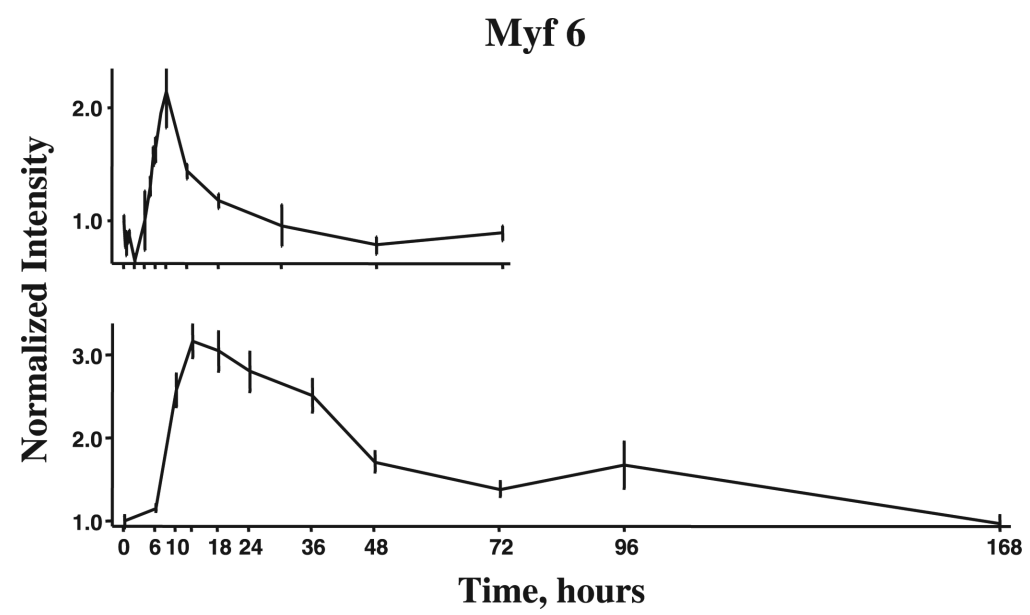

Fig. 14.

Response profiles of myogenic factor-6 (Myf6), which shows complex regulation of expression following acute (top) and chronic (bottom) MPL dosing. In the acute profile, there is an initial downregulation followed by a period of enhanced regulation. 

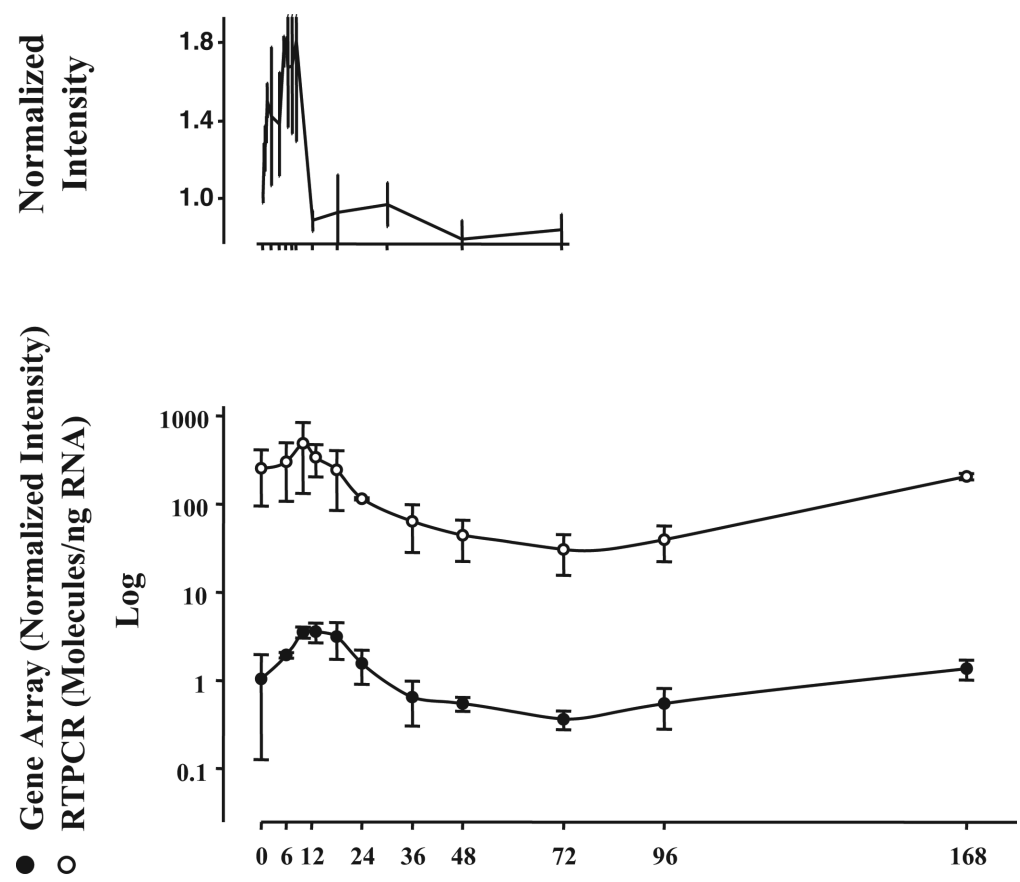

Time, hours

Fig. 15.

Response profiles of myostatin following acute dosing as determined from gene arrays (top) and following chronic dosing (bottom) as determined by both gene arrays ( $)$ and by kinetic-based quantitative RT-PCR $(\mathrm{O})$. 
MURF 1

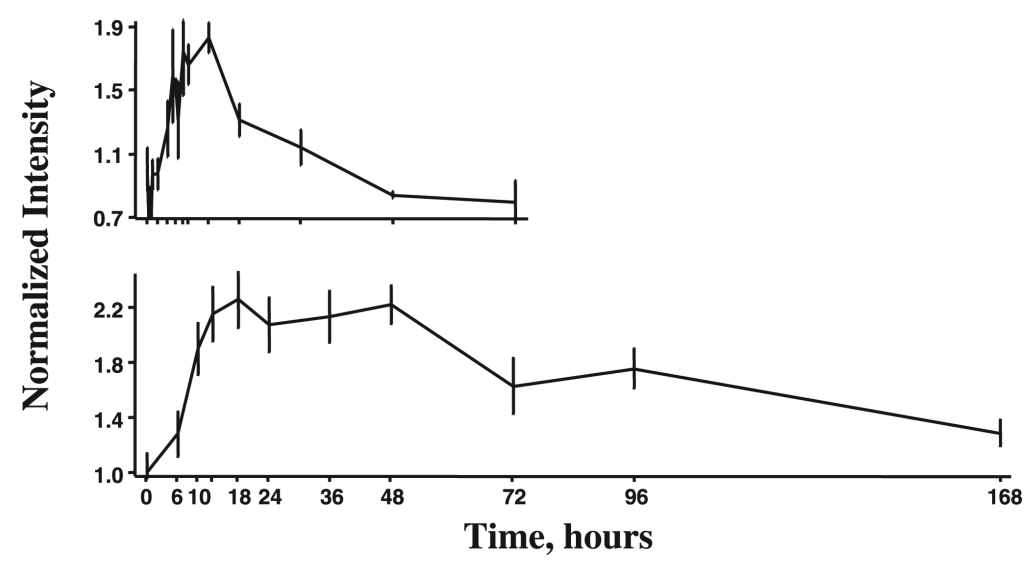

Fig. 16.

Response profiles of ring finger protein-28 (MURF1) following acute (top) and chronic (bottom) dosing. 


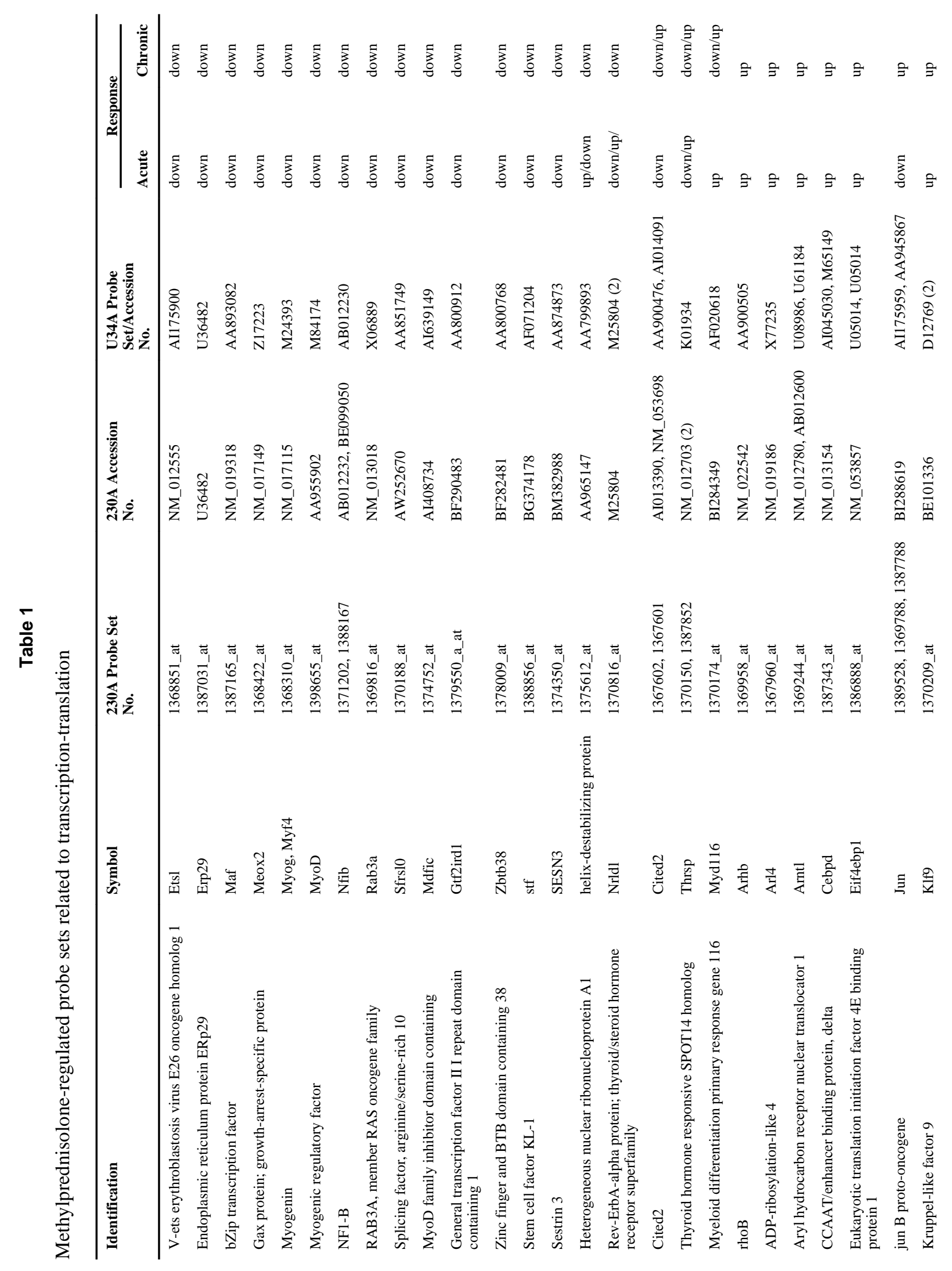

Physiol Genomics. Author manuscript; available in PMC 2014 October 06. 


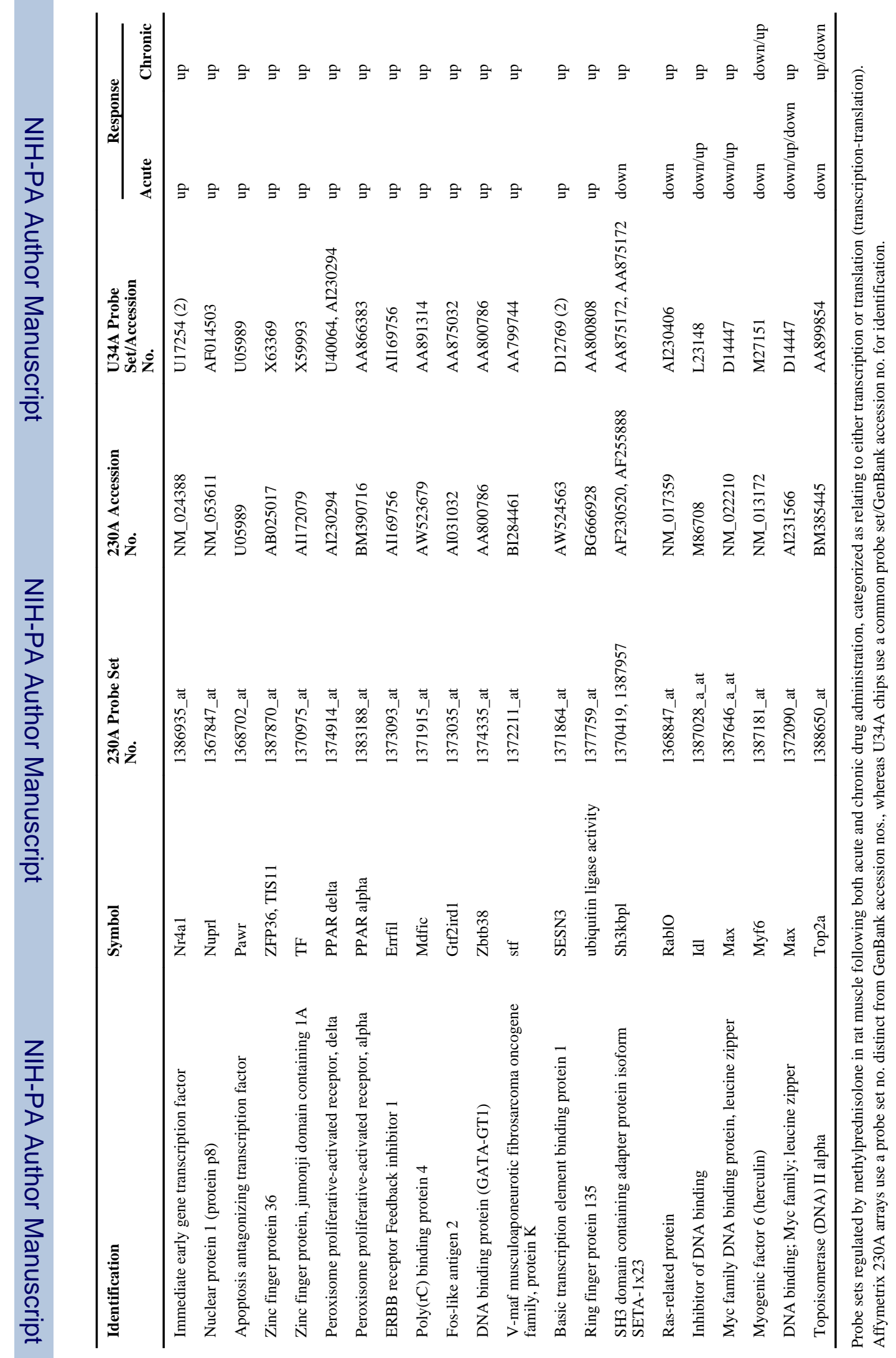




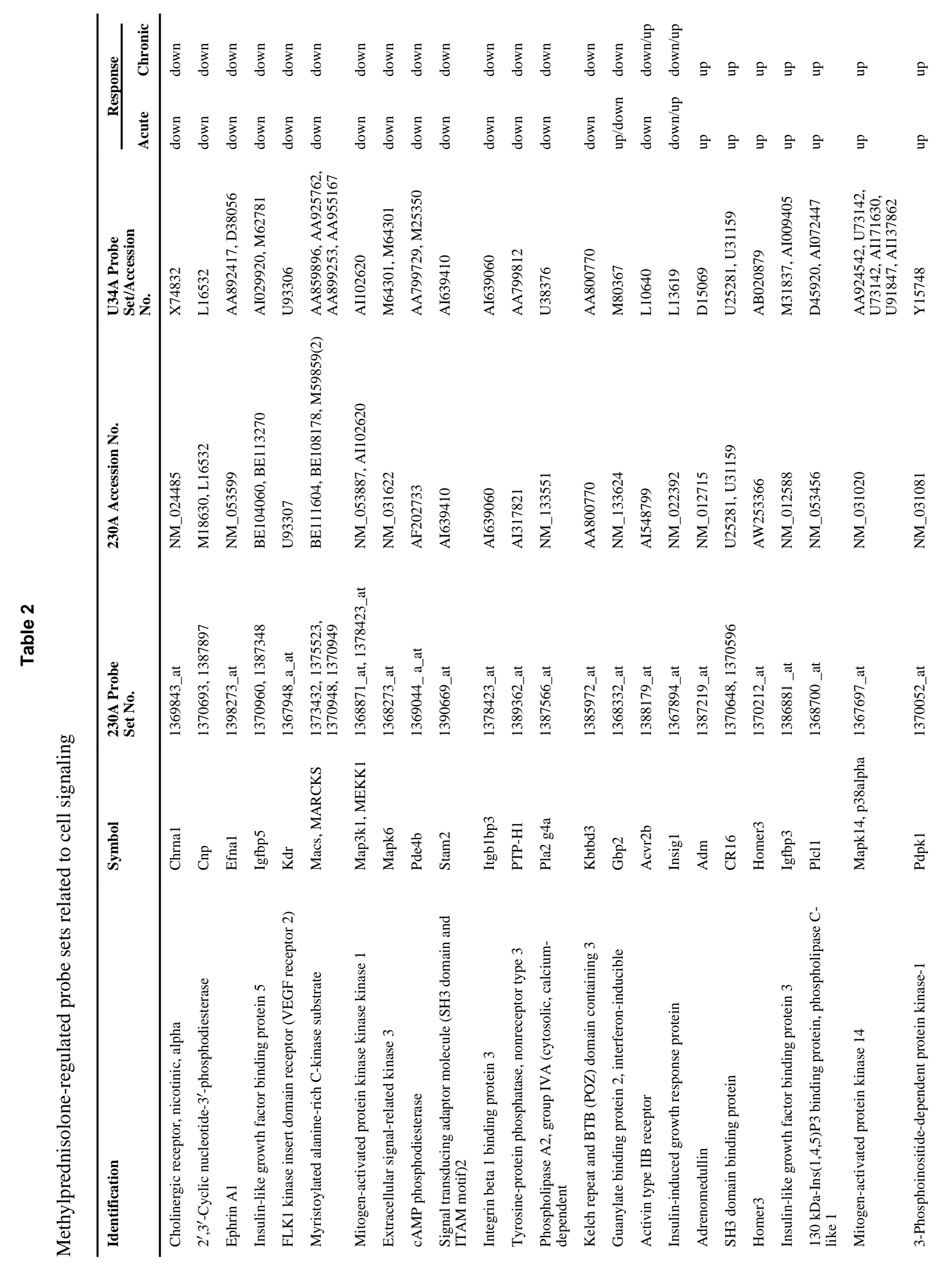




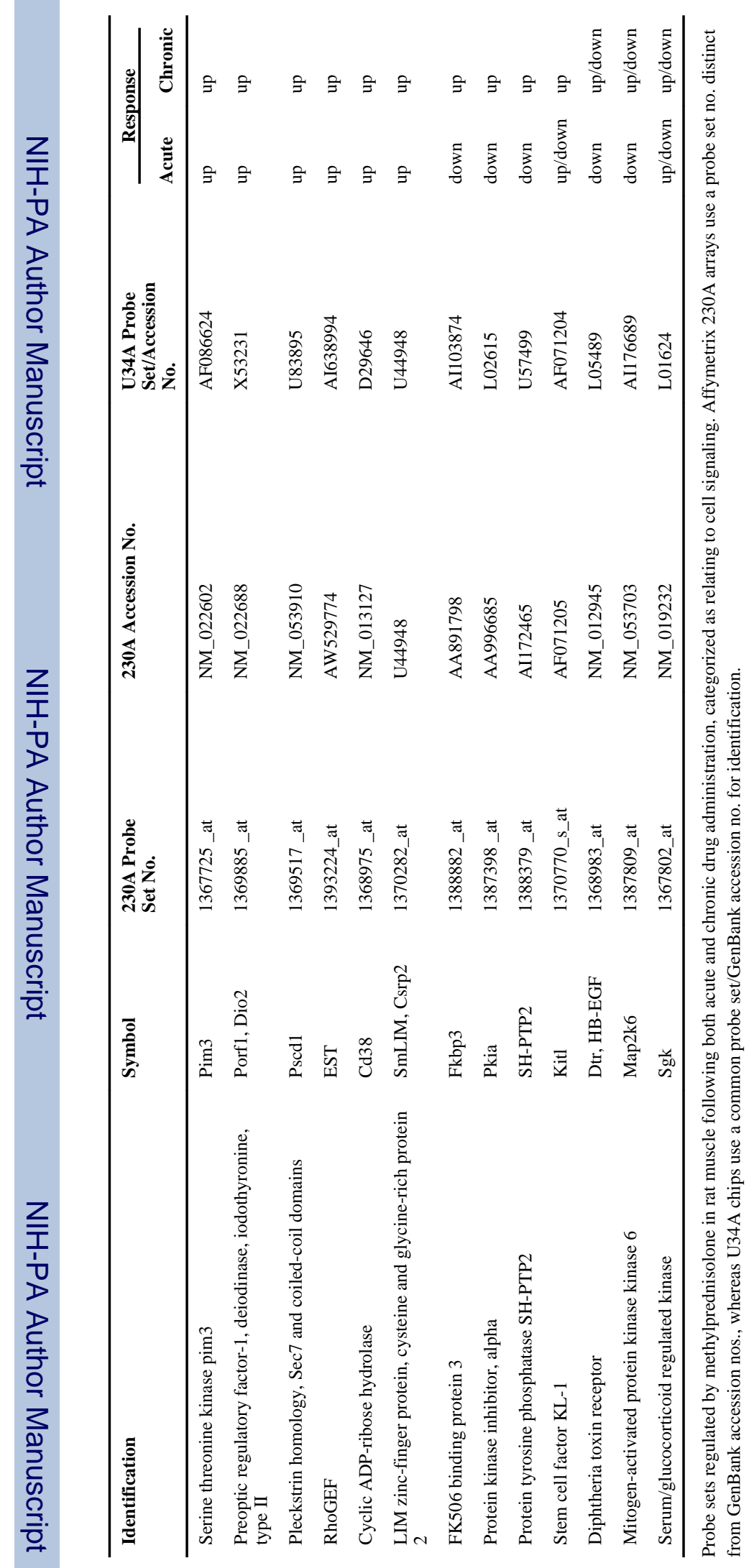




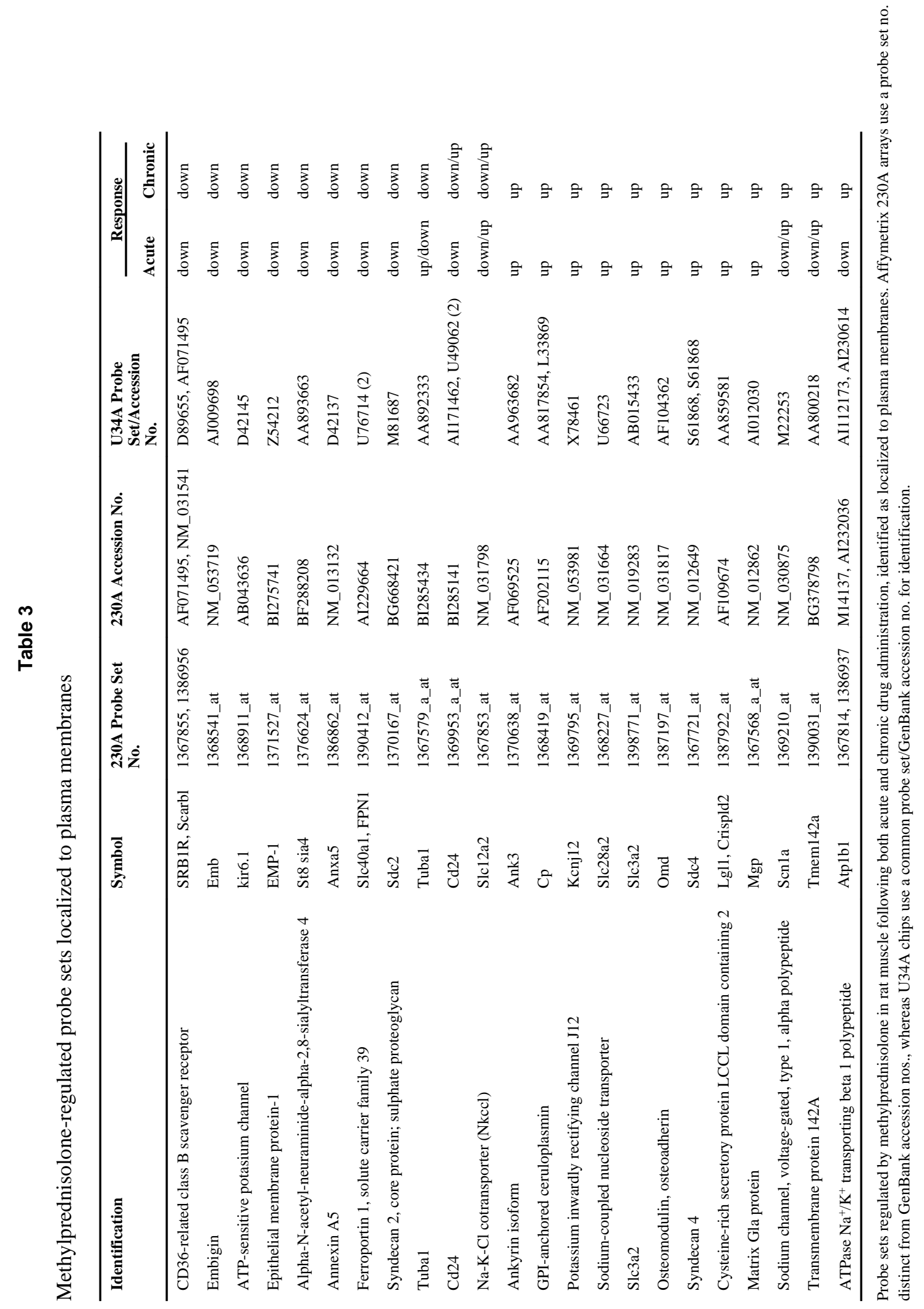


Table 4

Immune-related methylprednisolone-regulated probe sets

\begin{tabular}{|c|c|c|c|c|c|c|}
\hline \multirow[t]{2}{*}{ Identification } & \multirow[t]{2}{*}{ Symbol } & \multirow{2}{*}{$\begin{array}{l}\text { 230A Probe } \\
\text { Set No. }\end{array}$} & \multirow[t]{2}{*}{ 230A Accession No. } & \multirow{2}{*}{$\begin{array}{l}\text { U34A Probe Set/ } \\
\text { Accession No. }\end{array}$} & \multicolumn{2}{|c|}{ Response } \\
\hline & & & & & Acute & Chronic \\
\hline Latexin & Lxn & 1367768_at & NM_031655 & $\mathrm{X} 76985$ & down & down \\
\hline 11/3R gene; $\mathrm{MHC}$ & RT1Aw2 & 1371171_at & M10094 & X67504 & down & down \\
\hline $\begin{array}{l}\text { Major histocompatibility } \\
\text { complex class II }\end{array}$ & MHC class 2 & 1370383_s_at & BI279526 & X53054 & down & down \\
\hline Interleukin-33 & IL33 & 1389581_at & BF390510 & AA892986 & down & down \\
\hline $\begin{array}{l}\text { Chemokine (C-X-C motif) } \\
\text { ligand } 2\end{array}$ & Cxcl2 & 1368760_at & NM_053647 & U45965 & up & down \\
\hline $\begin{array}{l}\text { Vascular cell adhesion } \\
\text { molecule-1 }\end{array}$ & Vcam1 & 1368474_at & NM_012889 & M84488 & up/down & down \\
\hline Platelet factor 4 & Pf4 & 1371250_at & AI169104 & AI169104 & down & down \\
\hline $\begin{array}{l}\text { Interferon regulatory factor } \\
7\end{array}$ & Irf7 & 1383564_at & BF411036 & AA799861 & up/down/up & down/up/down \\
\hline $\begin{array}{l}\text { Arachidonate 5- } \\
\text { lipoxygenase activating } \\
\text { protein }\end{array}$ & Alox5ap & 1369672_at & NM_017260 & X52196 & up & up \\
\hline $\begin{array}{l}\text { B-cell translocation gene } 2 \text {, } \\
\text { anti-proliferative }\end{array}$ & Btg2 & 1386994_at & NM_017259 & M60921 & up & up \\
\hline $\begin{array}{l}\text { Complement component } 1 \text {, } \\
\text { q subcomponent, beta }\end{array}$ & $\mathrm{C} 1 \mathrm{qb}$ & 1370215_at & AW434057 & X71127 & up & up \\
\hline $\begin{array}{l}\text { Cytokine-inducible } \mathrm{SH} 2 \\
\text { containing protein }\end{array}$ & Cish & 1388233_at & AF065161 & AF065161 & up & up \\
\hline $\begin{array}{l}\text { T-cell receptor signal } \\
\text { transduction }\end{array}$ & Lnk & 1367723_a_at & NM_031621 & AA943555, U24652 & up & up \\
\hline Interferon gamma receptor & Ifngr & 1369956_at & NM_053783 & U68272 & up & up \\
\hline $\begin{array}{l}\text { Interferon-related } \\
\text { developmental regulator } 1\end{array}$ & Ifrd1 & 1367795_at & NM_019242 & AI014163 & up & up \\
\hline IL-15 & IL-15 & 1368375_a_at & AF015718 & AF015719 & up & up \\
\hline Interleukin 6 receptor & $\mathrm{Il} 6 \mathrm{r}$ & 1386987_at & NM_017020 & M58587 & up & up \\
\hline Lipocalin 2 & $\operatorname{Lcn} 2$ & 1387011_at & NM_130741 & AA946503 & up & up \\
\hline
\end{tabular}

Probe sets regulated by methylprednisolone in rat muscle following both acute and chronic drug administration, categorized as immune related. Affymetrix 230A arrays use a probe set no. distinct from GenBank accession nos., whereas U34A chips use a common probe set/GenBank accession no. for identification. 


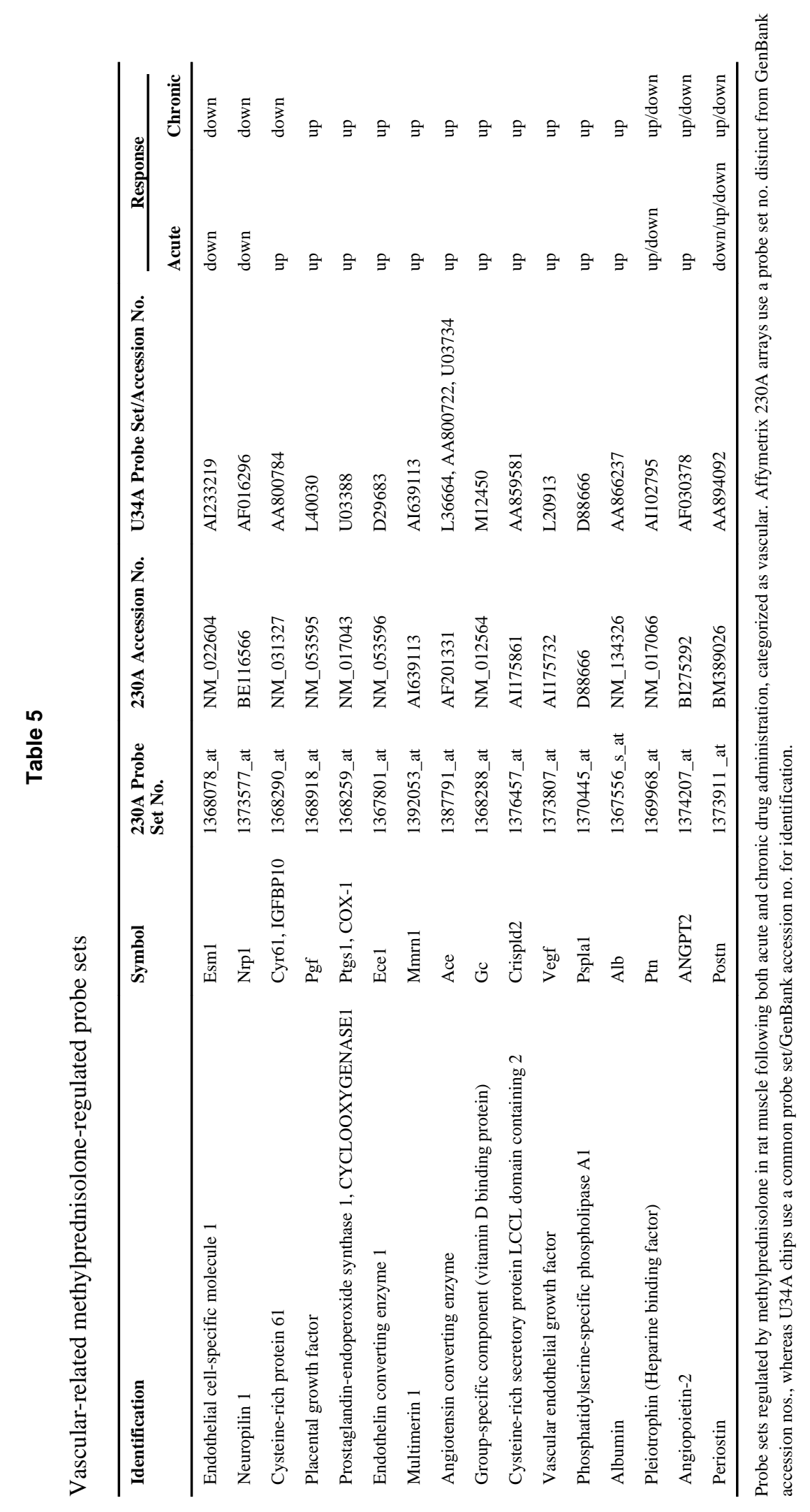

Physiol Genomics. Author manuscript; available in PMC 2014 October 06. 


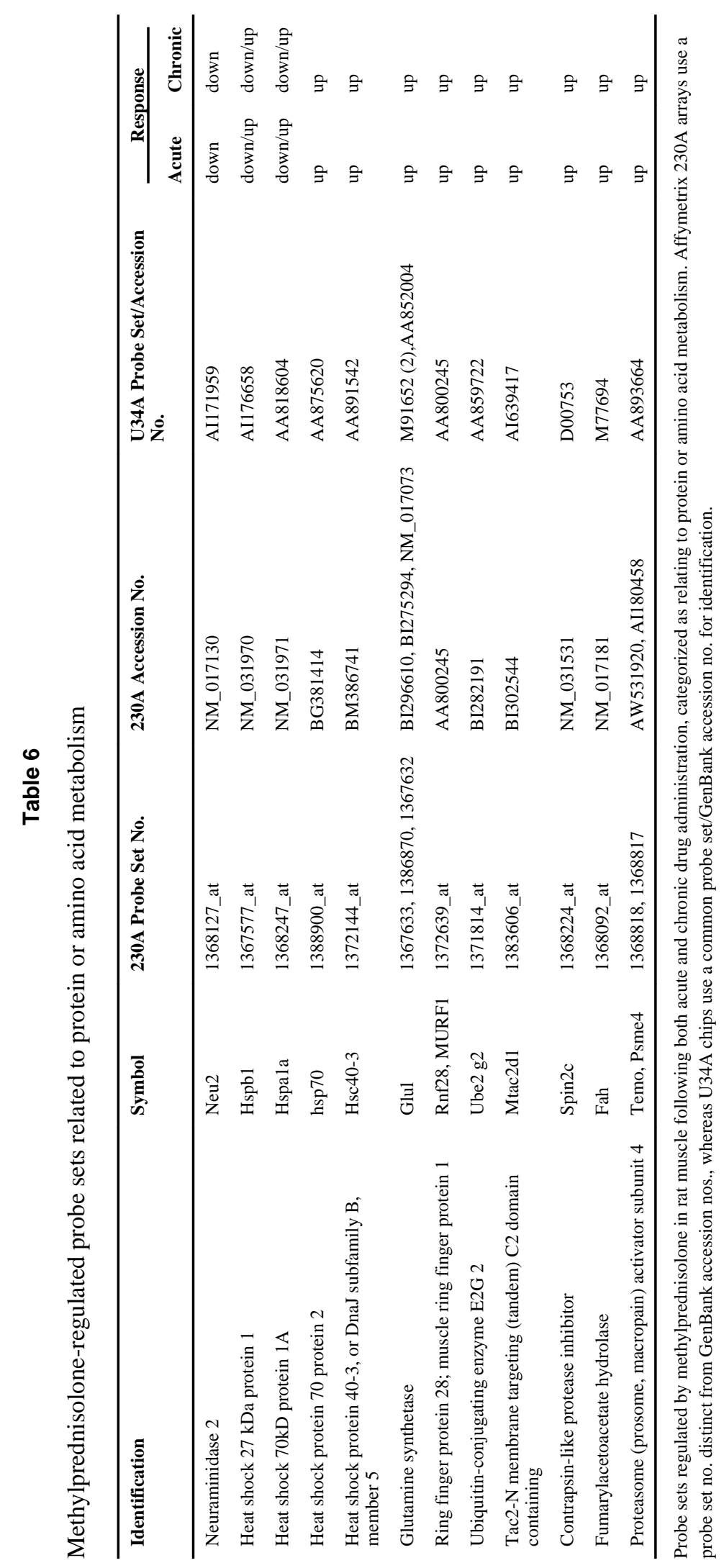

Physiol Genomics. Author manuscript; available in PMC 2014 October 06. 
Table 7

Nuclear-encoded mitochondrial genes regulated by methylprednisolone

\begin{tabular}{|c|c|c|c|c|c|c|}
\hline \multirow[t]{2}{*}{ Identification } & \multirow[t]{2}{*}{ Symbol } & \multirow{2}{*}{$\begin{array}{l}\text { 230A Probe } \\
\text { Set No. }\end{array}$} & \multirow[t]{2}{*}{ 230A Accession No. } & \multirow{2}{*}{$\begin{array}{l}\text { U34A Probe Set/ } \\
\text { Accession No. }\end{array}$} & \multicolumn{2}{|c|}{ Response } \\
\hline & & & & & Acute & Chronic \\
\hline Creatine kinase & $\mathrm{Ckb}$ & 1367740_at & M14400 & M57664 & down & down \\
\hline Diacylglycerol kinase zeta & Dgkz & 1367745_at & NM_031143 & D78588 & down & down \\
\hline L-arginine: glycine amidinotransferase & Gatm & 1367627_at & NM_031031 & U07971 & down & down \\
\hline Glycerol-3-phosophate dehydrogenase 2 & Gpd2 & 1369666_at & BG378763 & X78593 & down & down \\
\hline $\begin{array}{l}\text { NADH:ubiquinone oxidoreductase } \\
\text { MLRQ subunit }\end{array}$ & - & 1389018_at & BF411239 & AA686870 & down & down \\
\hline Microsomal glutathione-S-transferase 1 & Mgst1 & 1367612_at & NM_134349 & J03752 & down & down \\
\hline Cytochrome c, somatic & Cycs & 1387773_at & NM_012839 & K00750 & down & up \\
\hline $\begin{array}{l}\text { Mitochondrial cytochrome P-450 } \\
(\mathrm{P} 450 \mathrm{C} 27)\end{array}$ & CYP27 & 1387914_at & M73231 & Y07534, M38566 & up & up \\
\hline Uncoupling protein 3 & Ucp3 & 1387681_at & U92069 & AF030163, AF035943 & up/down & up \\
\hline Pantothenate kinase 2 & Pank2 & 1374541_at & BM392117 & AA891438 & down & up \\
\hline
\end{tabular}

Probe sets regulated by methylprednisolone in rat muscle following both acute and chronic drug administration, identified as nuclear-encoded mitochondrial genes. Affymetrix 230A arrays use a probe set no. distinct from GenBank accession nos., whereas U34A chips use a common probe set/GenBank accession no. for identification. 


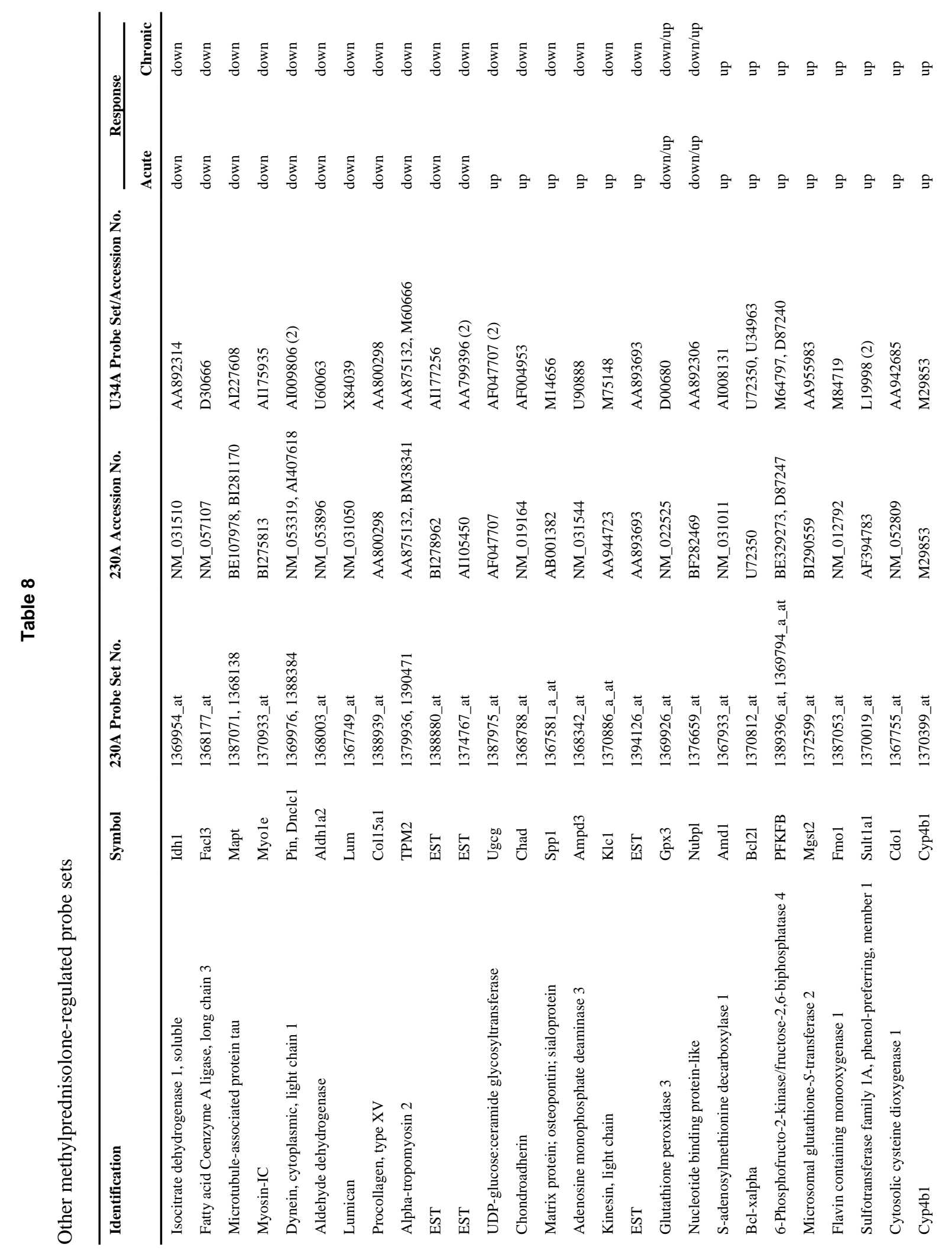

Physiol Genomics. Author manuscript; available in PMC 2014 October 06. 


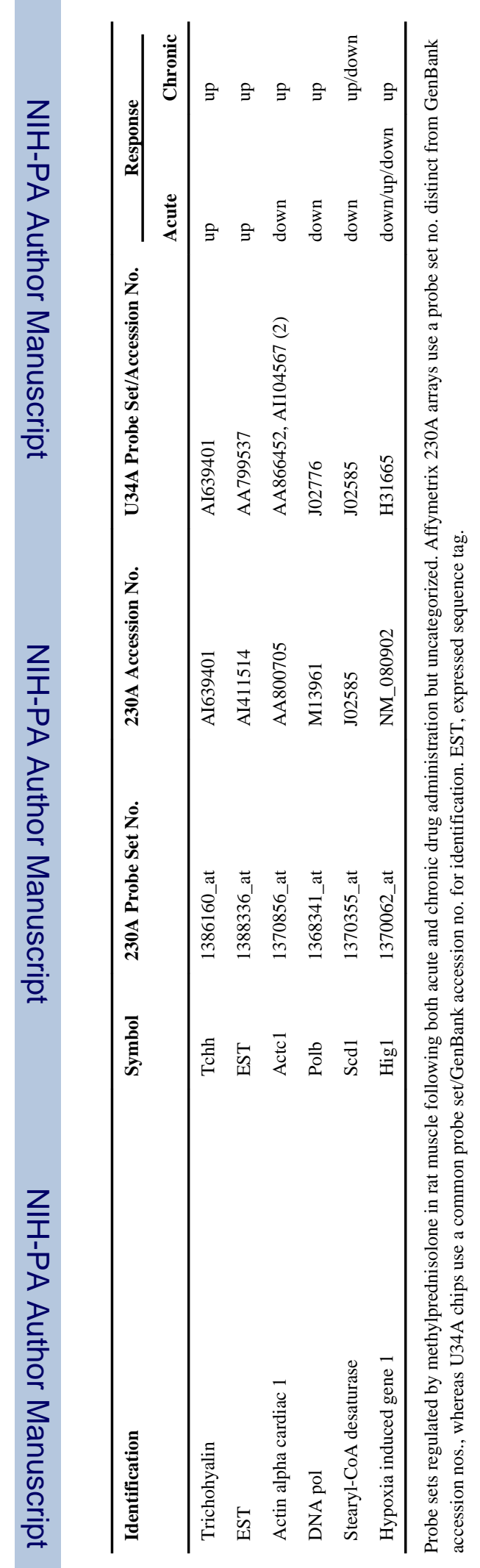

